\title{
Mixing and Reactive Fronts in the Subsurface
}

Rolle, Massimo; Le Borgne, Tanguy

Published in:

Reviews in Mineralogy and Geochemistry

Link to article, DOI:

10.2138/rmg.2018.85.5

Publication date:

2019

Document Version

Peer reviewed version

Link back to DTU Orbit

Citation (APA):

Rolle, M., \& Le Borgne, T. (2019). Mixing and Reactive Fronts in the Subsurface. Reviews in Mineralogy and Geochemistry, 85(1), 111-142. https://doi.org/10.2138/rmg.2018.85.5

\section{General rights}

Copyright and moral rights for the publications made accessible in the public portal are retained by the authors and/or other copyright owners and it is a condition of accessing publications that users recognise and abide by the legal requirements associated with these rights.

- Users may download and print one copy of any publication from the public portal for the purpose of private study or research.

- You may not further distribute the material or use it for any profit-making activity or commercial gain

- You may freely distribute the URL identifying the publication in the public portal

If you believe that this document breaches copyright please contact us providing details, and we will remove access to the work immediately and investigate your claim. 
This is a Post Print of the article published online $1^{\text {st }}$ Septembe 2019 in Reviews in Mineralogy and Geochemistry. The publishers' version is available at the permanent link:

http://dx.doi.org/10.2138/rmg.2018.85.5

\title{
Mixing and Reactive Fronts in the Subsurface
}

\author{
Massimo Rolle \\ Department of Environmental Engineering, Technical University of Denmark, Lyngby, Denmark \\ masro@env.dtu.dk
}

\section{Tanguy Le Borgne}

Geosciences Rennes, University of Rennes 1, Rennes, France

tanguy.le-borgne@univ-rennes1.fr 


\section{INTRODUCTION}

The interplay between mixing and reactive processes plays a pivotal role in a range of biogeochemical processes controlling the transport, transformation and turnover of chemical elements (McClain et al. 2003, Dentz et al. 2011, Valocchi et al. 2018). The study of these processes is of primary importance in many scientific disciplines and technical applications, including fluid mechanics, geochemistry, chemical engineering, reservoir engineering, subsurface and contaminant hydrology, water treatment, and remediation of contaminated sites. Mixing fronts often act as hot spots of biogeochemical reactions as they induce chemical disequilibrium and bring together reactants that may be otherwise segregated. By definition, reactive mixing fronts are characterized by strong and evolving chemical gradients; therefore, they cannot be in general understood and modeled under the conventional assumption of well-mixed chemical systems.

In this chapter, we provide an overview of some recent advances in the characterization, understanding and modeling of mixing and reactive fronts in the subsurface. We focus on incompressible water flow in porous media and we discuss two main types of mixing fronts, which play an important role in a range of applications from contaminant transport to geochemistry and eco-hydrology: (i) transient mixing fronts, where an advancing fluid progressively displaces a fluid of different chemical composition, (ii) steady-state mixing fronts, which develop around continuously released plumes. We present the basic mechanisms that govern the effective mixing and reaction rates of such fronts, and discuss recent findings that have changed our view on the deformation dynamics of these fronts in heterogeneous porous media and motivated the development of new modeling frameworks. We provide examples and discuss implications of mixing front dynamics for different biogeochemical reactions at scales ranging from the pore scale to the field scale.

Figure 1 illustrates a few examples of mixing and reaction fronts, which occur naturally (e.g., river/groundwater, sea/groundwater interactions) or as a result of contaminant release in groundwater from anthropogenic and/or natural sources. As they bring together reactants and create chemical disequilibrium, mixing fronts are often viewed as hot spots of biogeochemical reactions, where reaction rates can be much larger than in other areas (McMahon 2001, MartinezLanda et al. 2012). They can thus control key geochemical processes at interfaces between different water bodies (groundwater, rivers, lakes, oceans) (McClain et al. 2003, McAllister et al. 2015, Stegen et al. 2015, Hester et al. 2017), determine the fate of released pollutants and the delivery of reactants and amendments (Kitanidis and McCarty 2012). 


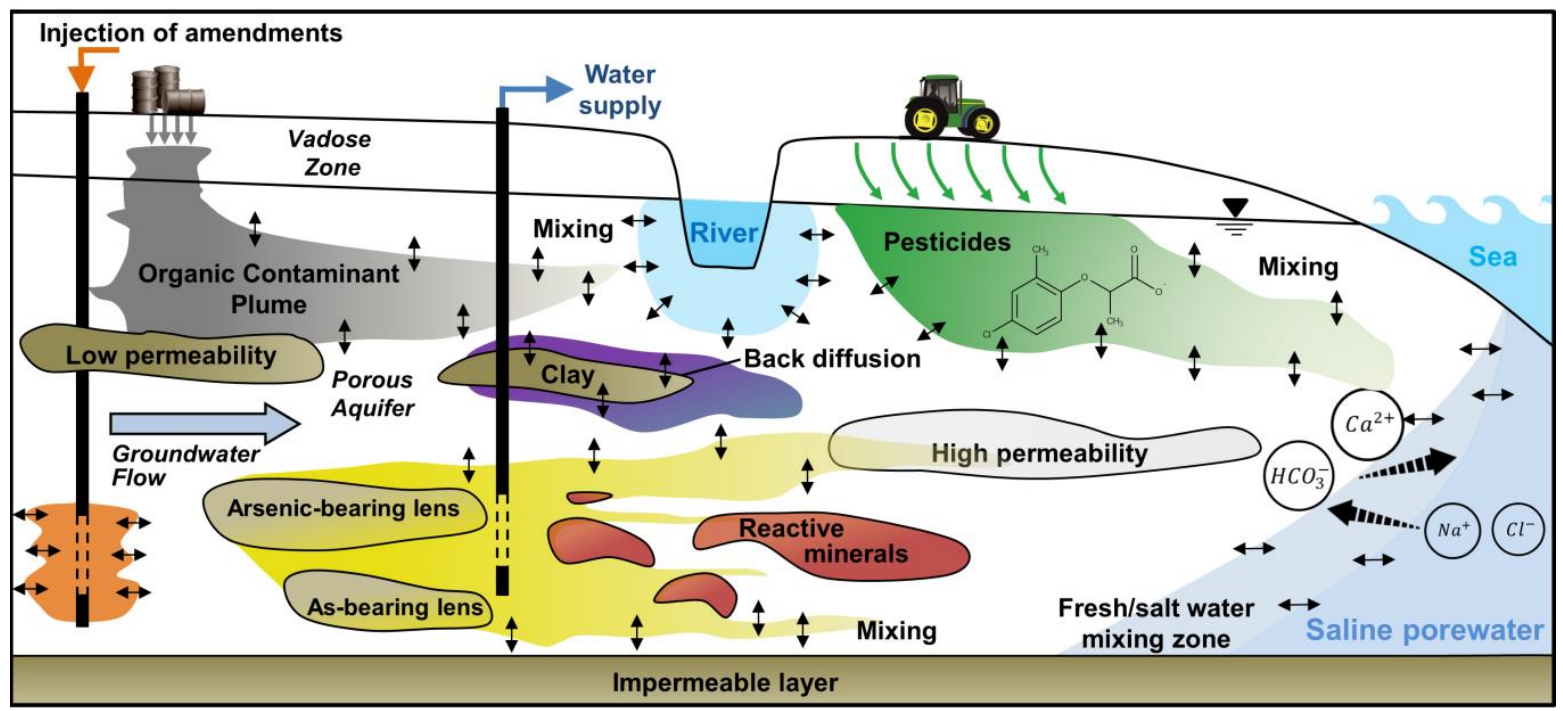

Figure 1. Schematic of groundwater contamination and mixing processes in the subsurface.

In the context of contaminant hydrology, hot spots of mixing occur at the fringes of contaminant plumes where the dissolved contaminants contact clean groundwater. The mixing of different waters triggers important reactions that may lead to contaminant degradation and to the alteration of the groundwater and/or porous medium chemistry through precipitation and dissolution reactions. For instance, the fate of organic contaminant plumes depends on microbially-mediated redox reactions in which the pollutants can be degraded through different electron acceptor processes (Chapelle 1995; Christensen et al. 2001). In the mixing zones at the plume fringes favorable conditions occur since soluble electron donors and acceptors, such as the organic contaminants and dissolved oxygen, nitrate and sulfate present in the pristine groundwater, come into contact (Anneser et al. 2008; Rolle et al. 2008). This favors the degrading activity of indigenous microorganisms that can access the necessary substrates for their metabolic activity.

Subsurface porous media are poorly mixed environments in which water flow is dominated by viscous forces and mixing is hindered by the absence of turbulence. Under such conditions of creeping flow, dilution of conservative solutes (i.e., their distribution over increasingly larger volumes or water fluxes) and mixing of initially segregated reactants control many reactive processes. These processes often represent the bottleneck and the rate-limiting steps for the overall reaction rates since they are typically slow and characterized by long time scales. Aqueous diffusion of the solutes in the pore water is the ultimate control on mixing processes since diffusion is the only physical mechanism leading to the increase of entropy and to the decrease of the peak concentration of solute plumes (Kitanidis 1994; Chiogna et al. 2011a). 


\section{Scales of mixing in porous media}

Mixing processes occur at different spatial and temporal scales. Figure 2 illustrates three important spatial scales of investigation of solute transport and mixing in porous media: the pore scale in solid-liquid domains with typical length of $\sim 10^{-2} \mathrm{~m}$, the laboratory or Darcy scale with length of common laboratory experimental setups $\left(\sim 10^{0} \mathrm{~m}\right)$, and the field scale $\left(\sim 10^{2} \mathrm{~m}\right)$.

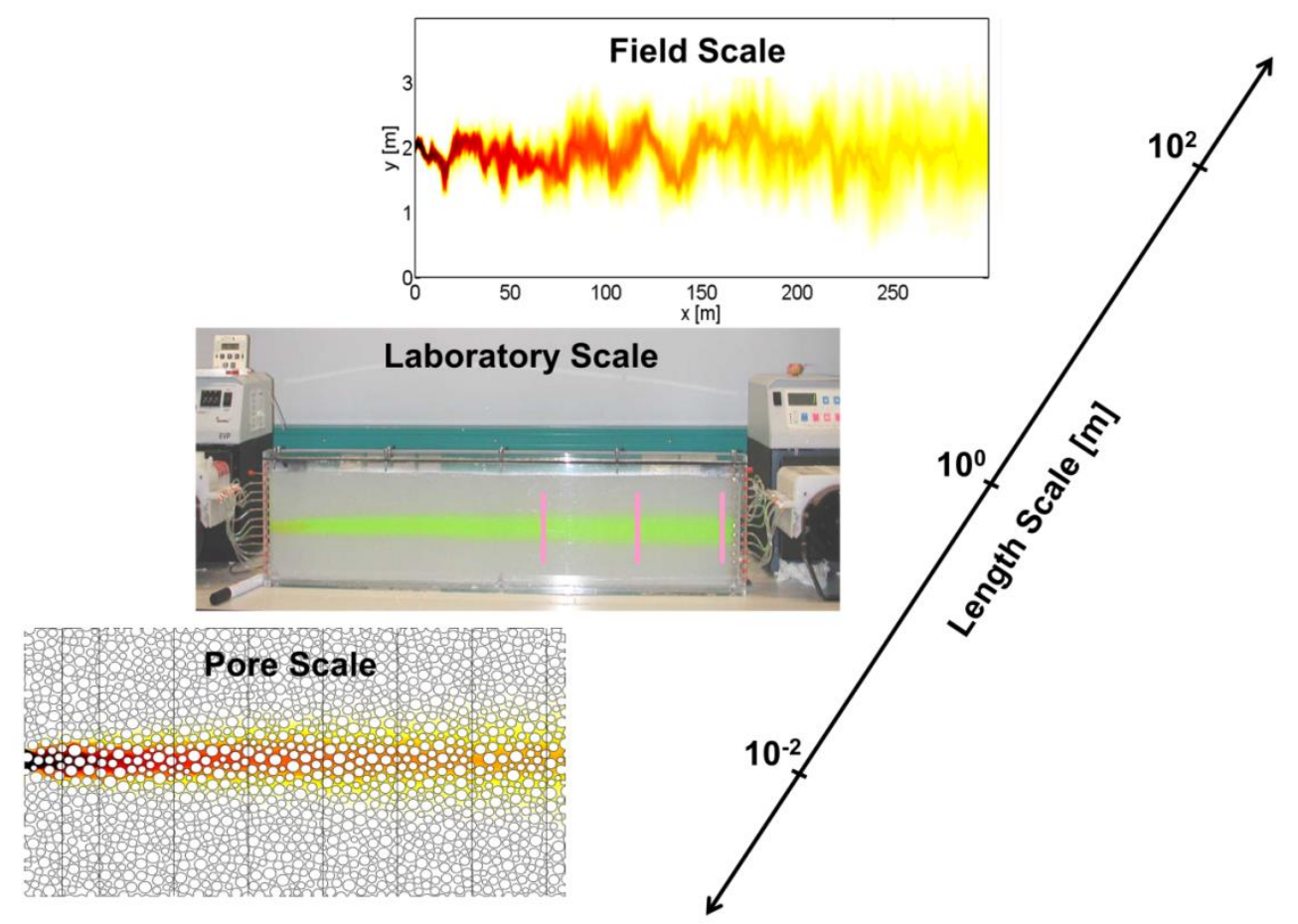

Figure 2. Scales of investigation of transport and mixing in the subsurface: examples showing continuously injected plumes at the pore, laboratory, and field scales (modified after Rolle et al. 2013a).

The study of flow, solute transport, and mixing at the pore scale has been instrumental to illuminate the coupling between different physical and reactive processes, to help gaining a fundamental understanding of these mechanisms, and to allow the interpretation of macroscopic experimental observations. At this scale the pore geometry is known and the flow problem is described by the Stokes equation and the continuity equation. The Stokes equation is a simplification of the NavierStokes equation for an incompressible fluid, disregarding inertia effects. Such momentum-flux terms are negligible in typical porous media flows, which are characterized by low Reynolds numbers. Considering steady-state conditions, the Stokes equation is a balance between potential energy and viscous dissipation terms:

$$
-\rho g \nabla \varphi+\mu \nabla^{2} \boldsymbol{u}=0
$$

where $\rho$ is the fluid density, $g$ is the constant of acceleration due to gravity, $\varphi$ is the hydraulic head $(p /(\rho g)+z), p$ is the pressure, $z$ is the elevation, $\mu$ is the dynamic viscosity, and $\boldsymbol{u}$ is the velocity vector. The continuity equation expresses the fluid mass conservation and can be written as: $\nabla \cdot \boldsymbol{u}=0$.

Transport at the pore scale is governed by the fundamental processes of advection, diffusion, and chemical reactions. The governing transport equation expresses the mass balance for a given dissolved aqueous species with concentration $C_{i}$ : 


$$
\frac{\partial C_{i}}{\partial t}+\nabla \cdot\left(\boldsymbol{u} C_{i}\right)-\nabla \cdot\left(D_{a q, i} \nabla C_{i}\right)=r_{i}
$$

where $\boldsymbol{u}$ is the velocity field determined from the solution of the flow problem, $D_{a q, i}$ is the aqueous diffusion coefficients of species $i$, and $r_{i}$ is the reactive term.

Microfluidics experiments and pore-scale modeling have been the main approaches to investigate flow and transport at the pore scale. The investigation has covered a wide variety of subsurface processes including conservative and reactive transport (Willingham et al. 2008; Hochstetler and Kitanidis 2013; Crevacore et al. 2016), longitudinal and transverse mixing (Acharya et al. 2007; Rolle et al. 2012; Hochstetler et al. 2013; Rolle and Kitanidis 2014; de Anna et al. 2014a), displacement and dissolution of non-aqueous phases (Chomsurin and Werth 2003; Bandara et al. 2013), bioreactive transport and growth of microbial populations (Knudson et al. 2007; Zhang et al. 2010a; Tartakovsky et al. 2013), and precipitation /dissolution of reactive minerals (Li et al. 2008; Zhang et al. 2010b; Molins et al. 2012; Yoon et al. 2012).

Although pore-scale descriptions are intuitive and derived from first-principles, descriptions of flow and transport in porous media at larger scales require macroscopic representations based on upscaling. Already at the scale of a laboratory experiment, a detailed representation of a porous medium at the pore scale becomes infeasible or impractical due to the lack of information about the details of the geometry and structure of the grains and pores of the medium and to the large computational demand. Instead, the medium is treated as a "continuum" with properties and variables that change continuously from point to point. Upscaling techniques result in the macroscopic representation of fluid flow and solute transport in porous media from the detailed descriptions at the pore-scale (e.g., Whitaker 1999). Averaging over space and time scales in a representative elementary volume (REV) of the porous medium, containing a large number of grains and pores, allows the replacement of a solid-liquid domain with an equivalent continuum. Such coarse graining leads to gradually changing variables and to upscaled parameters, which are of key importance for describing flow and transport processes. The macroscopic, continuum description of fluid flow in a saturated porous medium can be written as:

$$
S_{s} \frac{\partial \varphi}{\partial t}-\nabla \cdot(\mathbf{K} \nabla \varphi)=W
$$

where $S_{s}$ is the specific storage, $\varphi$ is the hydraulic head, $\mathbf{K}$ is the hydraulic conductivity tensor, and $\mathrm{W}$ is a sink/source term.

The continuum-scale governing equation for solute transport is the advection-dispersion-reaction equation:

$$
\frac{\partial C_{i}}{\partial t}+\nabla \cdot\left(v C_{i}\right)-\nabla \cdot\left(\mathbf{D}_{\mathbf{i}} \nabla C_{i}\right)=r_{i}
$$

This equation appears formally similar to the transport description at the pore scale, however here the concentration is defined over a larger support volume and varies more gradually compared to the concentration of an aqueous species at the pore scale. In Eq. 4, v is the seepage velocity vector whose components are average values that, in homogeneous and mildly heterogeneous media, exhibit considerably lower fluctuations compared to the highly-changing velocity in individual pore channels $(\boldsymbol{u}) ; \mathbf{D}_{\mathbf{i}}$ is the hydrodynamic dispersion tensor which accounts for the inherently coupled effects of aqueous diffusivity and pore velocity fluctuations in the pore channels; $r_{i}$ is the reaction rate term that is now expressed as a function of the averaged continuum scale concentrations. 
Equations 3 and 4 are the classical approaches adopted to describe flow and transport in the subsurface both at the laboratory and at the field scale. The key challenge under field conditions is the heterogeneity of geologic media. Such heterogeneity poses particular problems in upscaling solute transport that is more impacted by small scale fluctuations compared to fluid flow. If it would be possible to resolve the heterogeneity of geologic formations at a sufficiently small scale, a local scale continuum description of solute transport would allow capturing the main features controlling plume migration and mixing, as well as to represent the often observed non-Fickian behavior of solute breakthrough in subsurface systems (Kitanidis, 2017). This is not always possible due to the complexity of the subsurface and to the practical feasibility and costs of high resolution characterization of aquifer systems. Therefore, efforts have been dedicated to the development of effective descriptions of solute transport including macrodispersion approaches (e.g., Dagan 1989), dual and multicontinuum approaches (e.g., Haggerty and Gorelick 1995), and continuum time random walk (e.g., Berkowitz et al. 2006). Investigation performed at wellinstrumented research sites has been fundamental for the development of new approaches and transport theories in hydrogeology. Notable examples are the long-term investigations at the Borden site (Canada) and at the Columbus site (MADE site, USA). Detailed investigation of the heterogeneity in these aquifers has been possible due to the extensive characterization and numerous studies performed at these sites over decades (Sudicky and Illman 2011; Zheng et al. 2011). The study of reactive transport and mixing at the field scale has focused on different aspects, including transport of organic compounds (e.g., Prommer et al. 2006; Amos et al. 2011), inorganic pollutants (e.g., Kocar et al. 2008; Ma et al. 2010; Fakhreddine et al. 2016), propagation of leachate plumes from leaking landfills (e.g., Rolle et al. 2008; Bjerg et al. 2011) and interpretation of isotopic signatures (e.g., Eckert et al. 2012; Van Breukelen and Rolle 2012; Druhan and Maher 2017).

\section{MIXING AND MIXING-CONTROLLED REACTIONS}

Dilution, or mixing of a non-reactive solute with the surrounding fluid, is the process by which the solute tends to occupy a larger volume. If a system is chemically or biologically reactive, mixing of initially segregated species brings these reactants in contact, thus allowing the reaction to proceed. The subsurface is typically a poorly mixed environment since the presence of creeping flow and slow diffusive transport in geologic formations strongly limits the effective distribution and contact of dissolved chemical species. Therefore, mass transfer limitations are ubiquitous in the subsurface and often determine the overall rate of degradation processes. The study of mass transfer-limiting processes and their coupling with chemical reactions is important to understand reactive transport and contaminant degradation since observations in porous media at different scales show that the reaction rates may be considerably smaller compared to well-mixed laboratory setups (Kapoor et al. 1997, Meile and Tuncay 2006, Li et al. 2008).

\section{Dispersion in porous media}

The investigation of hydrodynamic dispersion in porous media is a complex and fascinating topic that has attracted the interest of many researchers from various disciplines including chemical engineering, reservoir engineering and contaminant hydrology. Different theories, modeling approaches, and experimental methods have been proposed to study dispersion in porous media (Bear 1972, Gelhar 1987, Dagan 1989, Cushman 2013). In particular in the field of reactive transport, issues concerning solute dispersion have been recognized as major challenges and have fostered active research in the last decades. As recently discussed by Kitanidis (2017), the study and the communication of dispersion in hydrogeology is still an area posing important challenges among researchers and practitioners. 
Dispersion terms are introduced at the continuum scale (Eqn. 4) by averaging the velocity and concentration fluctuations that cannot be resolved at the scale of observation (e.g., Kitanidis 1992; Whitaker 1999). In typical applications of solute transport in the subsurface, dispersion is described with the Fickian model considering the dispersive flux proportional to the gradient of the resolved concentration at the scale of interest. The dispersion term is strongly anisotropic since solute dispersion is inherently different along the direction of the flow (longitudinal dispersion) and perpendicular to the flow (transverse dispersion). Hydrodynamic dispersion depends on the coupled effects of the fundamental transport mechanisms of advection and diffusion. Although these mechanisms act together and are difficult to separate, hydrodynamic dispersion coefficients are typically parameterized as additive contributions of a velocity-independent pore diffusion term and a mechanical dispersion term that depends on the average flow velocity (Bear 1972). In the classical description of dispersion commonly used in hydrogeology (Scheidegger 1961), the mechanical dispersion term is described based on a linear dependence on the seepage velocity and using dispersivity coefficients, assumed to be properties of the porous medium.

Dispersion processes have been studied over a wide range of scales. At the laboratory scale, experimental observations of solute transport in packed beds have provided important contributions to the understanding of hydrodynamic dispersion. Empirical correlations based on a wealth of experimental data (Guedes de Carvalho and Delgado 2005; Delgado 2006) and on results and upscaling of pore-scale simulations have been proposed to describe longitudinal and transverse dispersion. Although the relations proposed may differ in their dependencies and coefficients, many studies show that a linear or quasi-linear dependence on the velocity and the grain size describe well the mechanical dispersion term of the longitudinal dispersion coefficient (e.g., Bijeljic et al. 2004; Delgado 2006; Muniruzzaman and Rolle 2017). In the transverse direction, hydrodynamic dispersion is essentially "enhanced diffusion" through the velocity variability in the pore channels. Correlations that capture well the essence of local transverse dispersion have a mechanical dispersion term that is non-linearly related to the average flow velocity (Delgado 2006; Chiogna et al. 2010) and is dependent on the aqueous diffusion coefficient of the transported solutes (Rolle et al. 2012 and 2013b; Ye et al. 2015a).

Fewer studies have investigated hydrodynamic dispersion at the block scale, which can correspond to the size of a grid cell in a numerically discretized domain or the scale of the sampling grid at a site (e.g., Rubin et al. 1999; Wang and Kitanidis 1999; de Barros and Dentz 2016). Heterogeneity cannot be resolved within the block and its effects on solute transport need to be factored in blockscale dispersion coefficients. The dispersion coefficients are essentially determined by averaging the velocity and concentration fluctuations within the block. As illustrated by a recent numerical study (Lee et al. 2018), the values of such coefficients depend on the interplay between advection and diffusion, the permeability contrast, and the structure of the heterogeneity within the block. At the field scale, dispersion has been studied intensively to describe solute transport in porous media. Macrodispersion theories have been developed to describe solute transport in heterogeneous flow fields and have contributed to form the research area of stochastic hydrogeology (e.g., Gelhar and Axness 1983; Dagan 1989; Rubin 2003). Macrodispersion coefficients expressed as half the rate of increase of the second central moment of the solute concentration can be linked to the heterogeneity of the formation and have been shown to describe well the evolution of conservative plumes after sufficient time from their release (e.g., Freyberg 1986; Garabedian et al. 1991). However, macrodispersion coefficients describing the spreading of solutes plumes would lead to substantial overestimation of the overall reactions if applied to describe mixing-controlled reactive transport (e.g., Semprini et al. 1990). Several studies have also emphasized the importance of the order of ensemble averaging and proposed the use of effective dispersion coefficients instead of 
ensemble (macrodispersion) coefficients to alleviate this problem (e.g., Dentz and de Barros, 2015).

When dealing with solute transport in the subsurface it is necessary to separate the effects of spreading from mixing (Dentz et al. 2011). This task is not easy since these processes are closely coupled (Villermaux et al. 2019). To this end, stochastic-flux related approaches have been proposed to determine mixing relevant dispersion coefficients and to quantify the uncertainty of mixing and mixing-controlled reactions (Cirpka et al. 2011 and 2012). In the following, we provide an overview of some recent approaches developed to study mixing and mixing-controlled reactions in porous media.

\section{Mechanisms of mixing and mixing enhancement}

The study of mixing processes in porous media is of primary importance to understand reactive solute transport in the subsurface. In particular, due to the poor mixing in subsurface flow systems it is crucial to identify the mechanisms that can lead to mixing enhancement. The heterogeneity of geologic formation can create complex flows that can substantially modify and enhance the mixing behavior of solute plumes. In the following, we briefly illustrate some key mechanisms for mixing in porous media that arise from the coupling between complex flows, causing stretching and deformation of solute plumes, and small scale processes such as diffusion and local scale dispersion. Figure 3 illustrates three important flow patterns causing deformation of solute plumes in porous media.

(a) Shear flow

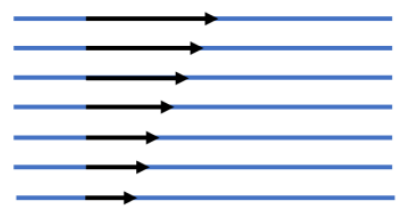

(b) Flow focusing and defocusing

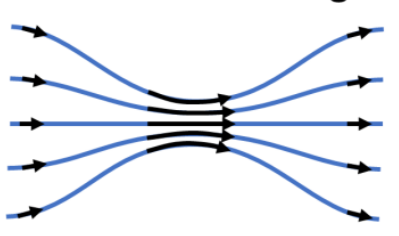

(c) Twisting flow

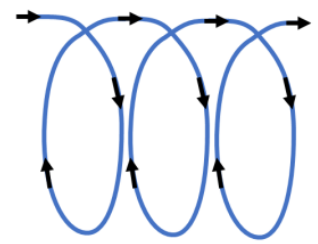

Figure 3. Example of flow patterns affecting mixing fronts and enhancing mixing and reaction rates in porous media: (a) shear flow results from differential velocities in neighboring streamlines; (b) flow focusing and defocusing results from variations of velocities along streamlines, which for incompressible fluids lead to contraction and divergence of streamlines; (b) twisting flow results from secondary motion in three-dimensional flow fields.

Front deformation and incomplete mixing. The elementary flow patterns illustrated in Fig. 3 are often combined to generate complex mixing dynamics at different scales. At the pore scale, the arrangement of solid grains and the pore space generates a broad velocity distribution (Bijeljic et al. 2011; de Anna et al. 2013; Kang et al. 2014; Holzner et al. 2015; Dentz et al. 2018) and complex streamline topologies (Lester et al. 2013), which leads to intricate mixing patterns (Fig. 4) (de Anna et al. 2014a,b; Jimenez et al. 2015, 2017; Lester et al. 2016). At field scale, structural heterogeneities, such as sedimentary layers and fractures, as well as non-uniform boundary conditions, also control the patterns of mixing between different solutes (de Barros et al. 2012; Engdahl et al. 2014; Le Borgne et al. 2014; Chiogna et al. 2015; Cirpka et al. 2015; Bandopadhyay et al. 2018). Stretching of mixing fronts by velocity gradients and streamline topology (Fig. 3) can greatly enhance mixing rates compared to diffusive mixing rates by increasing the area available for diffusive mass transfer and steepening concentration gradients (Ottino 1989; Chiogna et al. 
2011a, Le Borgne et al. 2014, Ye et al. 2015c).

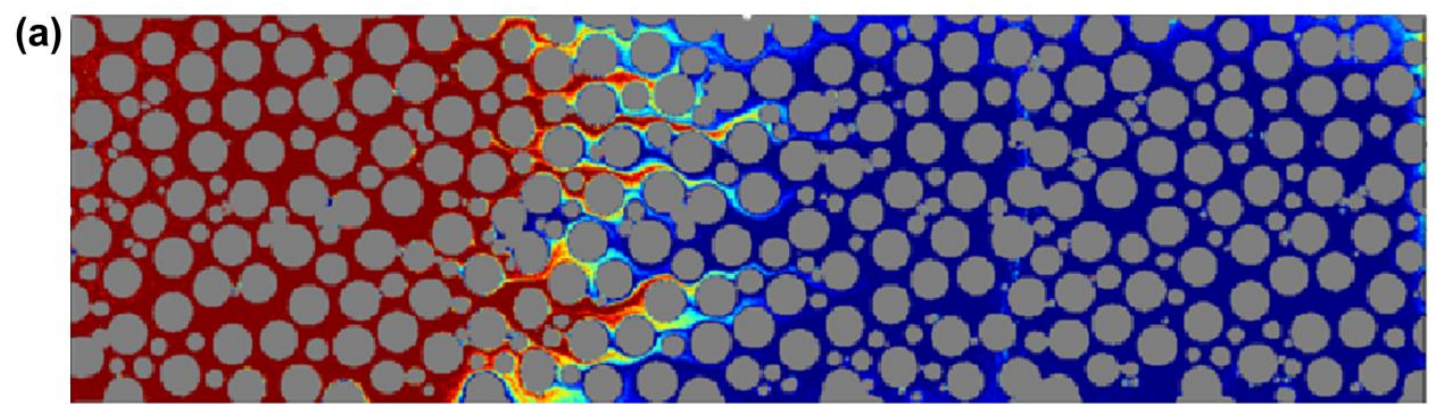

(b)

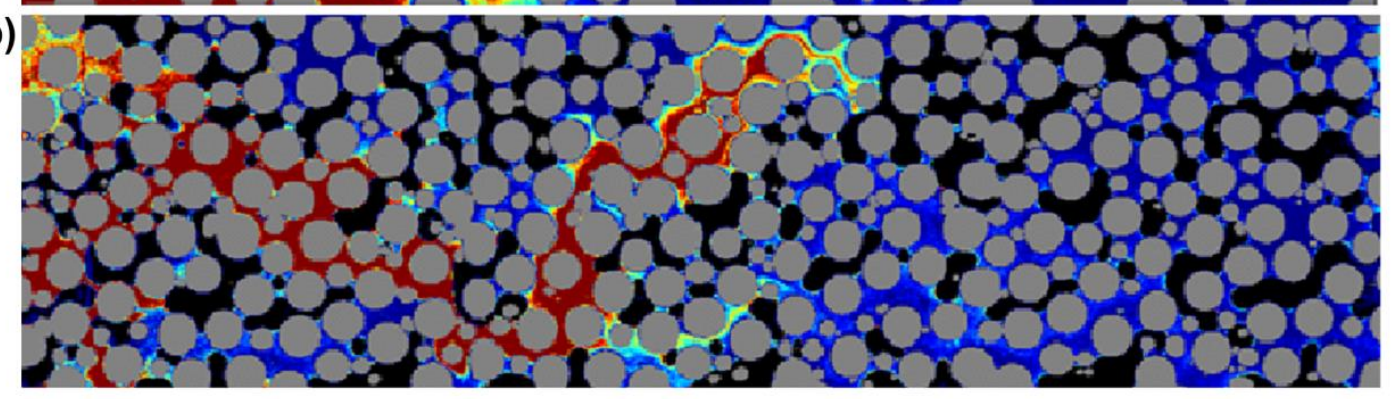

Figure 4. Conservative mixing experiments in 2D porous micromodels: (a) concentration field of a passive tracer front injected from left to right in a saturated porous medium (tracer concentration from red to yellow, water in blue and solid grains in grey); (b) concentration field of the same passive tracer injected in unsaturated conditions: the black zones represent air clusters (adapted from Jimenez et al. 2015, 2017).

The fingering pattern that develops in mixing fronts can also leave poorly mixed areas within the front (Fig. 4). Such incomplete mixing is the main reason why mixing and related chemical reactions are not accurately predicted in general by conventional dispersion theories assuming locally well-mixed conditions (Gramling, 2004, Le Borgne et al. 2011, de Anna et al. 2014b, Wright et al. 2017). While new fingers are continuously formed under flow, aggregation of solute fingers (de Anna et al. 2014a, Jimenez-Martinez et al. 2015) and diffusive mass transfer into immobile zones (Haggerty and Gorelick 1995; de Dreuzy et al. 2013; Babey et al. 2015) progressively reduce incomplete mixing. For saturated flows, de Anna et al. (2014b) have shown that the effective reaction rate becomes consistent with classical dispersion theory when solute fingers have aggregated as discussed in the following section. However, for unsaturated flows, Jimenez-Martinez et al. (2015) have demonstrated that the incomplete mixing regime is much longer as clusters of air prevent full aggregation of fingers (Fig. 4).

Shear flows. A simple deformation mechanism for mixing fronts is shear (Fig. 3a), which is created by a gradient of velocity in the direction transverse to the local flow (Ottino 1989, Bolster et al. 2011, Paster et al. 2015, Bandopadhyay et al. 2017, Souzy et al. 2018). In porous media, shear flows occur at multiple scales. At the pore scale, Poiseuille flow profiles, with zero velocity at the wall and maximum velocity at the pore center, act as shear flows in each pore. In Figure 4a, the deformation of the advancing front in finger patterns corresponds to a shear flow at pore scale where the velocity at the pore center is larger than at the walls. At larger scales, shear flows are created by permeability differences as illustrated in Figure 7 (Le Borgne et al. 2014, 2015). They can also be generated by non-uniform boundary conditions, even in homogeneous media. A hydraulic head gradient along the surface, leads to curved streamlines in the subsurface, on which the velocity decreases exponentially with depth, as occurs for instance in hyporheic flows and hillslopes (Toth 1963). This difference in velocities of neighboring streamlines acts effectively as a shear flow deforming advancing fronts of new water entering the subsurface (Bandopadhyay et 
al. 2018).

As the plumes advance in the domain, shear deformation increases the length of mixing fronts, thus enhancing mixing rates. In the simple shear flow scenario, where the velocity gradient is constant (Fig. 3a), the length of transient mixing fronts increases linearly in time (Bandopadhyay et al. 2017). In heterogeneous shear flows, where the shear rate is spatially distributed, the length of mixing fronts can follow a range of sub-exponential growth rates, including power law behaviors (Dentz et al. 2016). Under continuous solute injection, the differences in velocities in neighboring streamlines, driven by shear, affect the transient phase that leads ultimately to the steady concentration distribution and results in distinct solute residence times on each streamline, which may have important effects for different types of reactive processes

Flow focusing. In heterogeneous porous media, streamlines converge and diverge depending on the spatial distribution of the hydraulic conductivity (Fig. 3b). In high permeability zones (e.g., permeable sand and gravel sediments embedded in matrices with lower permeability) the flow is focused and streamlines converge as most of the water flux occurs through the highly permeable material. The outcomes of the simulations shown in Figure 5 illustrate this effect in 2-D cross sections at the laboratory and field scales. In the laboratory-scale domain three rectangular high$\mathrm{K}$ inclusions are embedded in a less permeable matrix (Fig. 5a). The flow is from left to right and the pattern of the computed streamlines clearly shows flow focusing into the high-K inclusions and defocusing downgradient of each inclusion. A similar but more complex pattern is observed in Figure 5b, where the streamlines are computed in a randomly generated binary field and the flow is focused into the irregularly shaped high permeability zones.

The focusing and defocusing of flow impacts solute transport and mixing in porous media since it affects: (i) the plume deformation, particularly relevant for transient fronts where the temporal dynamics of the front elongation can be related to the velocity distribution (Dentz et al. 2016); (ii) the residence time of a solute parcel within the inclusion is smaller than the time needed to cover the same distance in the low-K matrix; (iii) the distance between streamlines in the high$\mathrm{K}$ zones is reduced and the transverse concentration gradients are increased; thus, transverse mass transfer between streamlines occurs over a smaller distance inside the inclusions; (iv) the transverse dispersion coefficient is higher in the high-K zones than in the surrounding matrix due to the higher velocity and to the larger grain size of the more permeable material.

In a study of continuously injected plumes, Werth et al. (2006) showed that the overall results of flow focusing and defocusing is an enhancement of transverse mixing due to the effects of increased mass transfer and dispersion coefficients within the high-permeability inclusions. The same authors also derived analytical expressions to quantify mixing enhancement factors in 2-D setups and showed that flow focusing in high-permeability inclusions leads to significant mixing and reaction enhancement. The combined effect of dimensionality and flow focusing on mixing of groundwater plumes has been investigated in successive studies. Ye et al. (2015b) performed laboratory-scale experiments directly comparing mixing enhancement through flow focusing in quasi 2-D and fully 3-D porous media. Expressions for mixing-enhancement factors in 3-D domains were also derived. The experiments and the derived analytical expressions showed that although plume dilution is larger in 3-D setups due to the additional degree of freedom, stemming from the third spatial dimension that ensures an increased contact area between the plume and the surrounding groundwater (Kitanidis, 1994; Ye et al. 2015b), mixing enhancement is less effective in 3-D than in 2-D systems. Indeed, mixing enhancement in 2-D was shown to be the upper limiting case for fully 3-D cases. The study also demonstrated the importance of the parameterization of local transverse dispersion and showed that in the hypothetical case of pure velocity independent pore diffusion and identical flow focusing in both transverse directions no enhancement of transverse mixing would occur. Finally, both experimental and numerical studies in 2-D and 3-D 
setups have highlighted the importance not only of the permeability contrast but also of the spatial distribution of the high-permeability inclusions. For instance, the effect of flow focusing on mixing enhancement is particularly effective when permeable inclusions are located close to plume sources. In such conditions flow focusing of an "undiluted plume" with sharp concentration gradients at the fringes results in very effective mixing enhancement.
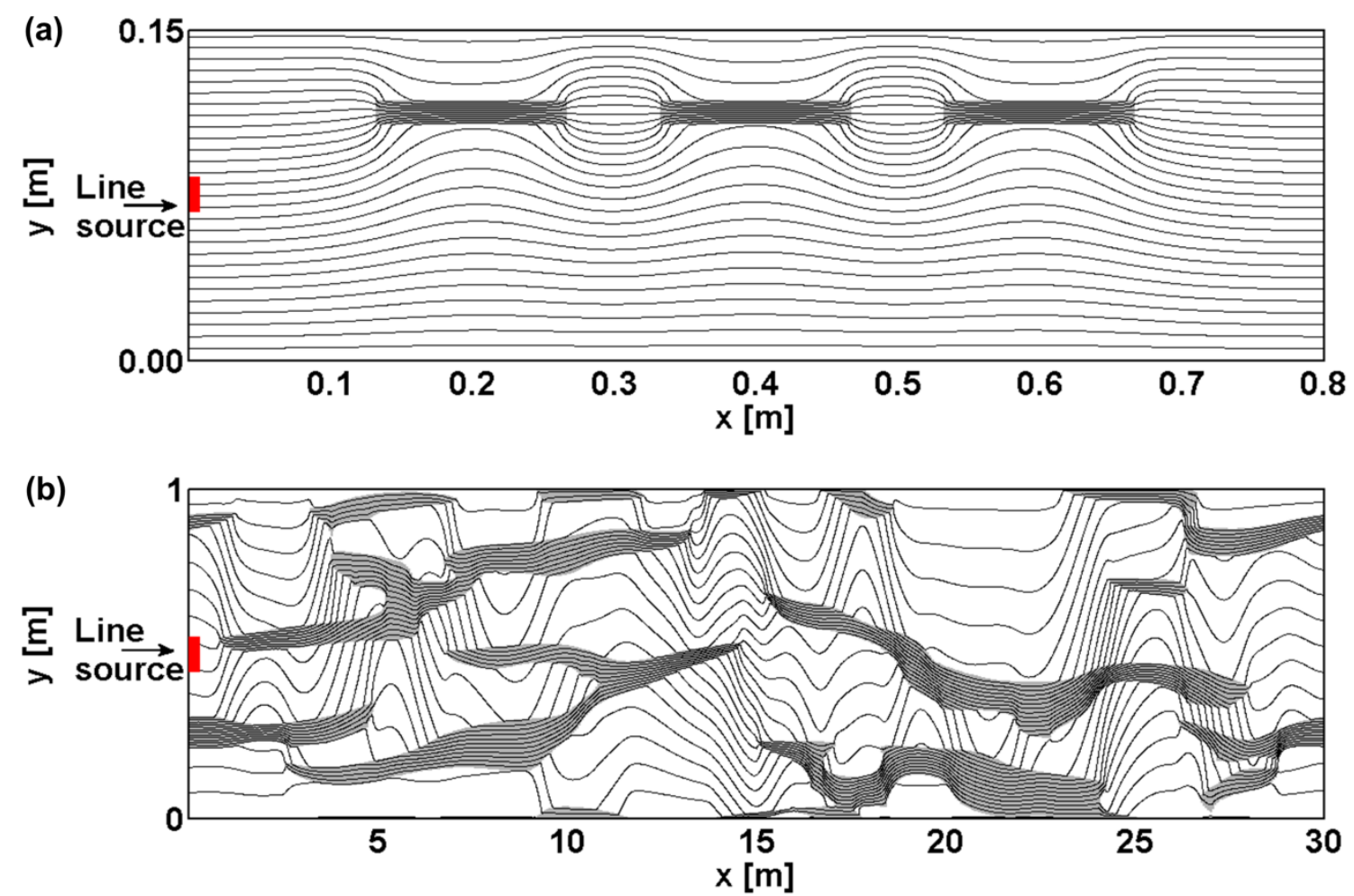

Figure 5. Flow focusing in heterogeneous porous media: streamlines computed for 2-D cross sectional domains at (a) laboratory scale and (b) field scale (from Chiogna et al. 2011a).

Twisting of streamlines. Streamlines in heterogeneous formations can exhibit complex patterns that can significantly impact the extent of mixing of solute plumes both under steady-state and transient conditions (e.g., Mays and Neupauer et 2012; de Barros et al. 2012; Trefry et al. 2012; Piscopo et al. 2013; Lester et al. 2013). Most studies have focused on two-dimensional setups probably due to the difficulty of running high-resolution three-dimensional numerical simulations and of performing three-dimensional flow-through experiments. However, 3-D systems are of great interest since in fully three-dimensional setups additional mechanisms can impact mixing dynamics. For instance, anisotropy of hydraulic conductivity can lead to groundwater whirls (Bakker and Hemker 2004; Stauffer 2007; Chiogna et al. 2014 and 2015). In 3-D heterogeneous anisotropic media secondary motion involving streamlines twisting, folding and intertwining can overlay the primary velocity field (Fig. 3c). The impact of these processes on mixing enhancement for continuously emitted plumes has been studied in detailed 3-D numerical simulations at the field scale (Cirpka et al. 2015). At the pore scale, the topology of threedimensional streamlines is expected to generate stretching and folding processes characteristic of chaotic mixing dynamics (Lester et al. 2013; Turuban et al. 2018). This leads to an exponential increase of mixing fronts, which is expected to dominate asymptotically the linear deformation created by shear (Lester et al. 2016).

Experimental evidence of the occurrence of streamline twisting and helical flow in porous 
media was provided by Ye et al. (2015c and 2016). Flow-through experiments were performed in a three-dimensional heterogeneous anisotropic porous medium consisting in alternating angled strips of coarse and fine materials in two different layers (Fig. 6a). Such architecture of the porous medium was chosen as a simplified representation of herringbone cross-stratification and entailed twisting of streamlines due to the alternating pattern and orientation of the high and low permeability zones (Fig. 6b). The twisting pattern of the streamlines strongly affects solute transport and mixing compared to an analogous homogeneous porous medium. Evidence of plume spiraling within the heterogeneous anisotropic medium was provided by using a dye tracer and by freezing and slicing the porous medium at different cross sections. The helical flow in the flowthrough setup causes the deformation of the material surface of the plume. This results in an increase of the diffusive and transverse dispersive fluxes and, thus, of mass exchange between the streamlines leading to considerable mixing enhancement.
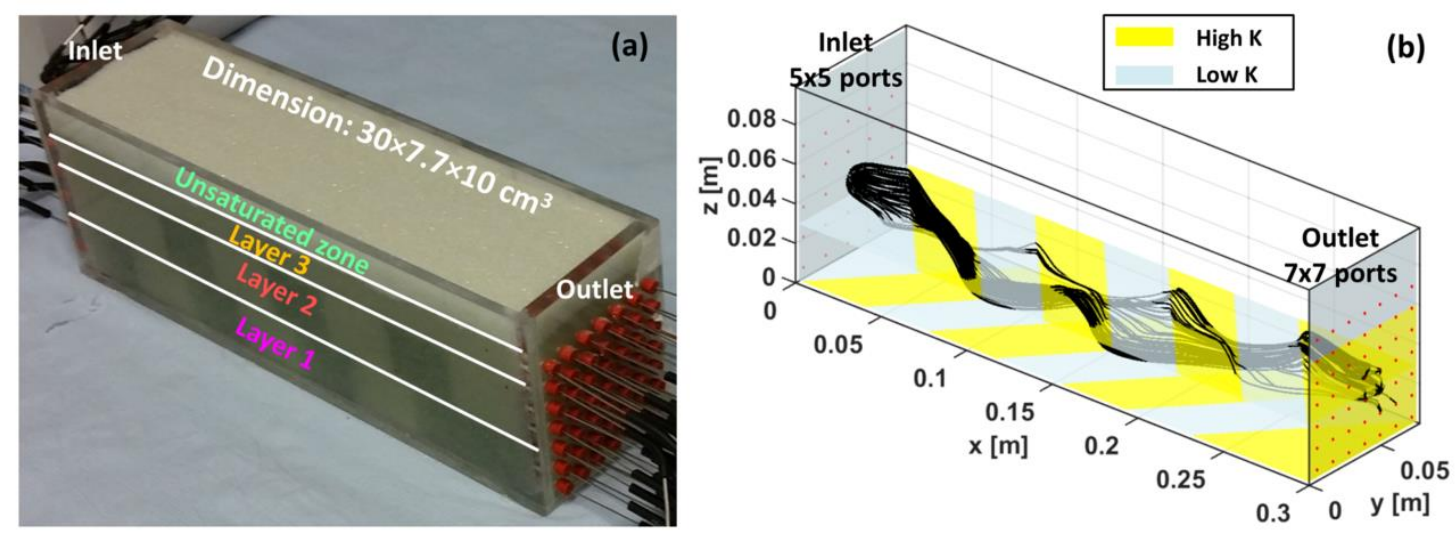

Figure 6. (a) 3-D experimental setup with a heterogeneous anisotropic porous medium and high-resolution sampling at the outlet; (b) twisting streamlines computed for the 3-D system (from Ye et al. 2016).

\section{Quantification of mixing}

The quantification of mixing and mixing enhancement in porous media has attracted considerable interest particularly in recent years as it became apparent the need to distinguish between plume spreading and mixing for transport of solute plumes in the subsurface (Kitanidis, 1994, Dentz et al. 2011, Chiogna et al. 2012, Le Borgne et al. 2015). Metrics used to quantify mixing for conservative and reactive transport include the dilution index (Kitanidis 1994; de Barros et al. 2012; Rolle and Kitanidis 2014; Di Dato et al. 2017), the scalar dissipation rate (De Simoni et al. 2005; Luo et al. 2008; Le Borgne et al. 2010; Engdahl et al. 2013), the concentration variance (Kapoor and Kitanidis 1996), and the gradient of the solute concentration square (Bolster et al. 2011; Chiogna et al. 2011b). In principle, most of these metrics can be derived from the knowledge of the full concentration probability density function (Le Borgne et al. 2015). In this section, we illustrate two approaches for the quantification of mixing in porous media. We first discuss the case of a transient mixing front advancing into a domain using the lamella theory of mixing. Successively, we present an analysis of mixing for continuously injected plumes using the dilution index.

Lamella theory of mixing. The lamella theory of mixing is a Lagrangian framework that links the distribution of stretching rates along mixing interfaces to mixing and reaction rates. In this framework, the mixing interface is discretized into a series of elements, which deform advectively according to the local velocity field (Fig. 7). These elements are called lamellae in 2D and sheets 
in 3D. While we present here the theory for the 2D case, its extension to 3D is discussed in details by Martínez-Ruiz et al. (2018). The lamella representation is based on the formulation of the transport equations (equation 2 at the pore scale or equation 4 at the Darcy scale) in a reference frame attached to the interface between the two fluids that mix (Fig. 7b). The first axis of the reference frame, corresponding to coordinate $\sigma$, is aligned with the lamella, while the second axis, corresponding to coordinate $n$, is oriented in the direction perpendicular to the lamella. Since concentration gradients are maximum along coordinate $n$, diffusive mass transfer along $\sigma$ can be neglected compared to diffusive mass transfer along $n$. Hence, the transport equation in this frame reduces to (Ranz 1979, Bandopadhyay et al. 2017 and 2018):

$$
\frac{\partial C_{i}}{\partial t}-\gamma n \frac{\partial C_{i}}{\partial n}-\frac{\partial}{\partial n}\left(D \frac{\partial C_{i}}{\partial n}\right)=r_{i}
$$

where $\gamma$ is the stretching rate, $D$ is the molecular diffusion coefficient at pore scale and the dispersion coefficient at Darcy scale, and $r_{\mathrm{i}}$ is the local reaction rate. The second term quantifies the effect of compression perpendicular to the direction of elongation in incompressible fluids and the third term quantifies the effect of diffusion.

(a)

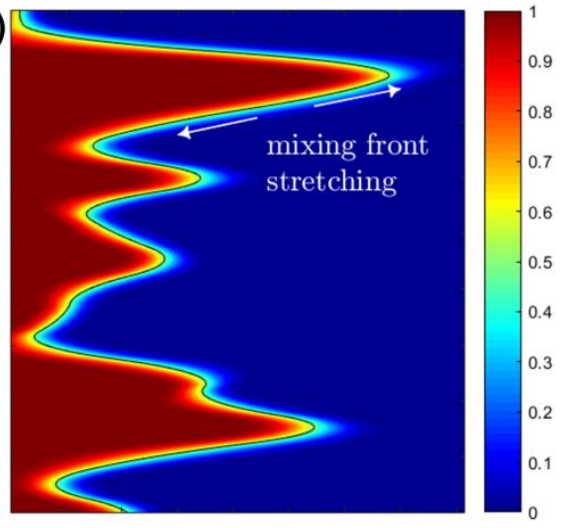

(b)

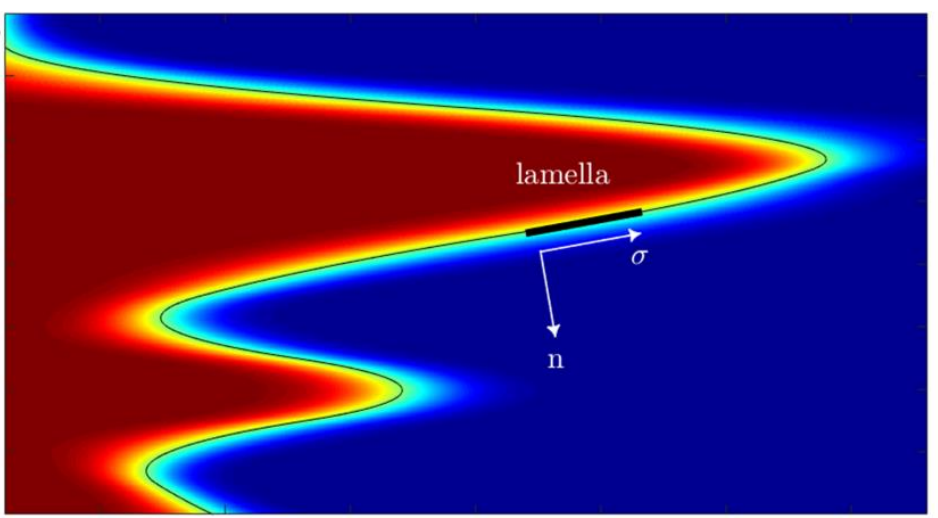

Figure 7. Illustration of the lamella description of mixing for a random shear flow. (a) Decomposition of a mixing front into a collection of lamellae, which quantify the local elongation (the color scale represents the concentration of the solute injected from the left, normalized to its maximum concentration); (b) zoom on a lamella showing the definition of the Lagrangian coordinate system attached to the lamella (adapted from Le Borgne et al. 2014).

Equation 5 can be used to derive analytical solutions for effective mixing and reactions rates in mixing fronts as a function of the stretching rate, which is defined for a lamella as:

$$
\gamma=\frac{1}{\rho} \frac{d \rho}{d t}
$$

where $\rho$ is the elongation, defined as $\rho=L / L_{0}$, with $L$ the current lamellae length and $L_{0}$ its initial length. The temporal evolution of the front elongation for the main types of deformation dynamics is given in Table 1. In uniform flows, there is no velocity gradient and the elongation is $\rho=1$. In radial flows, as occur for instance around injection wells, the elongation of an injected front evolves as the square root of time due to streamline divergence (Le Borgne et al. 2014). Shear flows (Fig. 3a) lead to a linear increase of elongation. As discussed above, chaotic flows are generated by stretching and folding processes in three-dimensional porous media both at the pore scale and in anisotropic porous media (Lester et al., 2013, 2016, Turuban et al. 2018, Ye et al. 2016). They can also be engineered designing transient pumping and injection schemes to enhance mixing (Piscopo et al. 2013). 
Elongation of mixing fronts increases the area available for diffusive mass transfer and enhances concentration gradients by compression in the direction perpendicular to the elongation direction. The mixing time is defined as the time at which compression balances with diffusion and concentration gradients cease to increase. It is therefore the time at which concentration gradients are maximum (de Anna et al. 2014a, Le Borgne et al. 2014, Le Borgne et al. 2015, Bandopadhyay et al. 2018, Villermaux et al. 2019). Expressions for the mixing time are given in Table 1 for the different elongation dynamics.

Table 1. Temporal evolution of the elongation $\rho$ for the main types of deformation and corresponding mixing times where compression and diffusion balance each other and concentration gradients are maximum.

\begin{tabular}{lcccc}
\hline & Uniform flow & Radial flows $^{\mathbf{a}}$ & Shear flows $^{\mathbf{b}}$ & Chaotic flows $^{\mathbf{d}}$ \\
\hline Elongation & $\rho=1$ & $\rho=\sqrt{\left(Q / \pi h \phi r_{w}^{2}\right) t}$ & $\rho=\nabla v t$ & $\rho=e^{\gamma t}$ \\
\hline \multirow{2}{*}{ Mixing time } & N/A & $\tau=s_{0} r_{w} \sqrt{\pi h \phi / D Q}$ & $\tau=\nabla v^{-1}\left(s_{0}^{2} \nabla v / D\right)^{1 / 3}$ & $\tau=\gamma^{-1} \ln \left(D / s_{0}^{2} \gamma\right)$ \\
\hline
\end{tabular}

a. for an injection into an aquifer/fracture $\mathrm{Q}$ is the flow rate, $\mathrm{h}$ is the vertical size of the permeable domain, $\phi$ is the porosity and $r_{w}$ is the well radius (Le Borgne et al. 2014), b. $\nabla v$ the is the velocity gradient (Bandopadhyay et al. 2017, 2018), c. the exponent $\alpha$ is related to the velocity distribution (Le Borgne et al. 2015), d. $\gamma$ is the Lyapunov exponent (Lester et al. 2013). In all cases $S_{0}$ is the characteristic initial width of the front, and D is the diffusion coefficient at the pore scale and the dispersion coefficient at Darcy scale.

For the conservative case, $r_{i}=0$, the solution of Equation (5) for a front is (Bandopadhyay et al. 2018):

$$
C(n, t)=\frac{C_{0}}{2}\left(1-\operatorname{erf}\left(\frac{n \rho}{s_{0} \sqrt{1+4 \theta}}\right)\right)
$$

with so the initial width of the front and $\theta$ the dimensionless time:

$$
\theta=\int_{0}^{t} d t D \rho^{2} / s_{0}^{2}
$$

To quantify the effect of front stretching on the enhancement of mixing rates, we use here the scalar dissipation rate as a metric of mixing (Le Borgne et al. 2010). In 2D, the scalar dissipation rate is defined as:

$$
\chi=\iint d x d y D(\nabla c)^{2}
$$

with $D$ the local diffusion/dispersion coefficient. As discussed in the next section, the interest of the scalar dissipation rate is that it is directly related to the reaction rate for fast equilibrium reactions (Le Borgne et al. 2010).

Using equation 7 , the effect of the front deformation on the effective mixing rate can be derived from the scalar dissipation rate as:

$$
\chi=\iint d n d l D(\nabla c)^{2}=\frac{D l_{0} c_{0}^{2}}{\sqrt{2 \pi} s_{0}} \frac{\rho^{2}}{\sqrt{1+4 \theta}}
$$


with $l_{0}$ the initial length of the total front. The enhancement of mixing by front deformation is illustrated in Fig. 8, where the mixing rate is computed from equation 10 for the case of a shear flow (Fig. 7 and Table 1). While the mixing rate decreases in time in the absence of shear, due to the diffusive decay of concentration gradients, it increases in time with shear as concentration gradients are sustained by the front elongation. Therefore, front deformation by shear flow can increase the mixing rate by orders of magnitude compared to non-deformed fronts. This approach can be extended to random velocity fields both at the pore scale (de Anna et al. 2014) and at the Darcy scale (Le Borgne et al. 2013, 2014, 2015; Bandopadhyay et al. 2018), and for continuously injected steady plumes (Lester et al. 2016). We discuss its application to reactive mixing in a latter section.

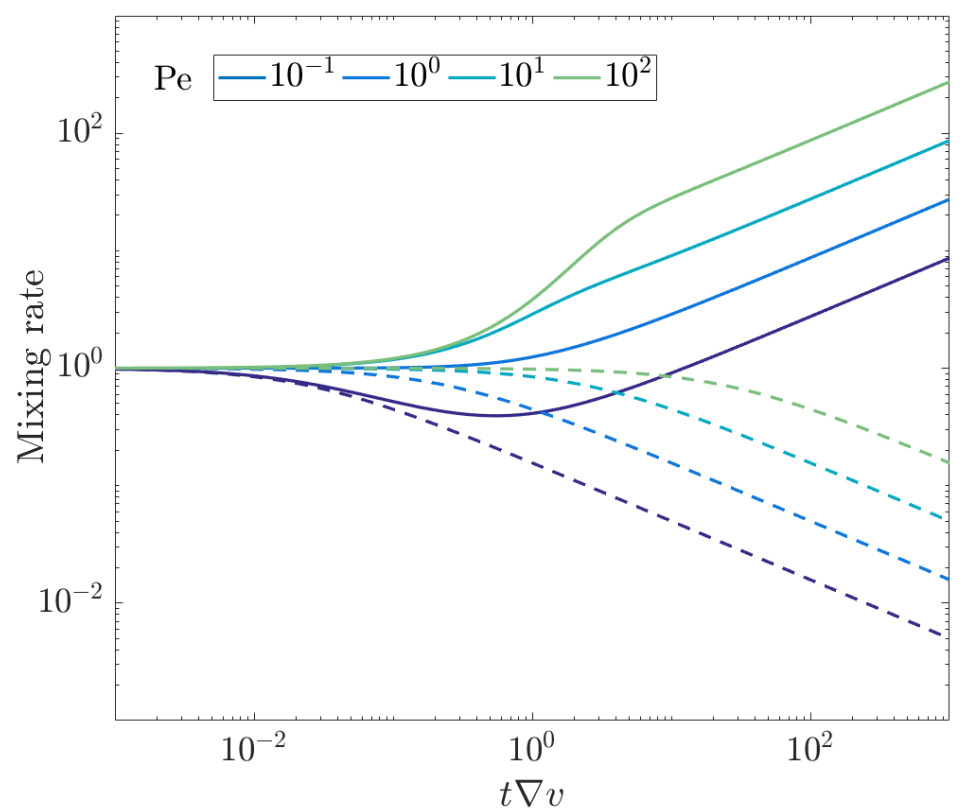

Figure 8. Temporal evolution of the mixing rate for different shear rates (continuous lines) compared to the mixing rates in the absence of shear (dashed lines). The mixing rate is defined as the scalar dissipation rate normalized by its initial value. These results were obtained assuming a constant diffusion coefficient (adapted from Bandopadhyay et al. 2018).

Analysis of continuously injected plumes with the flux-related dilution index. Here we illustrate a few examples of quantification of mixing and mixing enhancement for continuously emitted solute plumes using the concept of flux-related dilution index (Rolle et al. 2009). This metric is based on the volumetric dilution index introduced by Kitanidis (1994) but, instead of representing the distribution of a solute mass over an increasing volume, it quantifies dilution as the distribution of a given solute mass flux over a larger water flux. Physically, the flux-related dilution index represents the effective volumetric discharge transporting the solute flux at a given position along the main flow direction. In a bounded domain and for steady-state transport the flux-related dilution index is expressed as:

$$
E_{Q}(x)=\exp \left(-\int_{\Omega} p_{Q}(\boldsymbol{x}) \ln \left(p_{Q}(\boldsymbol{x})\right) q_{x}(\boldsymbol{x}) d \Omega\right)
$$

where $q_{x}$ is the component of the specific discharge normal to the cross-sectional area $\Omega$ and $p_{Q}(x)$ is the flux-related probability density function: 


$$
p_{Q}(\boldsymbol{x})=\frac{C_{i}(\boldsymbol{x})}{\int_{\Omega} C_{i}(\boldsymbol{x}) q_{x}(\boldsymbol{x}) d \Omega}
$$

As the volumetric dilution index, $E_{Q}(x)$ is based on the Shannon entropy and, for conservative transport under steady-state conditions, monotonically increases with the travel distance since transverse dispersion distributes the solute mass flux over a larger water flux. The flux-related dilution index is particularly suited to quantify mixing for continuously emitted solutes, whereas the volumetric dilution index allows the quantification of mixing when compounds are introduced as pulses in a flow-through domain. A transient flux-related dilution index has been more recently introduced to analyze the dilution breakthrough of solute slugs and to complement the analysis based on the volumetric dilution index (Rolle and Kitanidis, 2014). Figure 9 shows two examples of steady-state fluorescein plumes in a homogeneous and a heterogeneous porous media. In the heterogeneous setup two high-permeability inclusions cause focusing of the streamlines and of the dissolved plume and represent hot-spots for mixing enhancement.

(a)

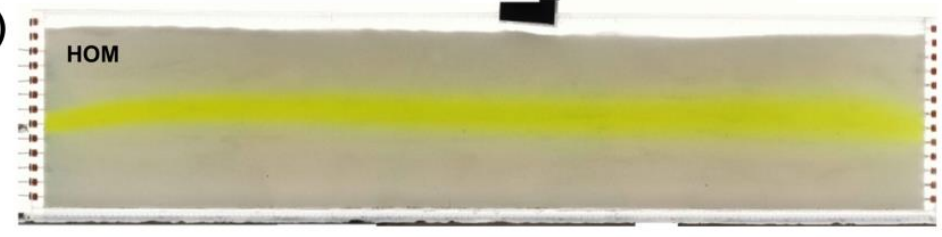

(b)

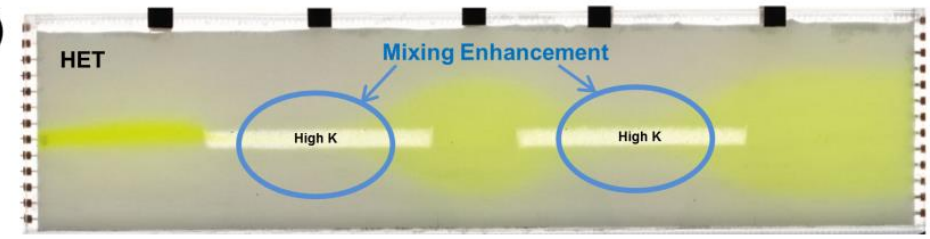

(c)

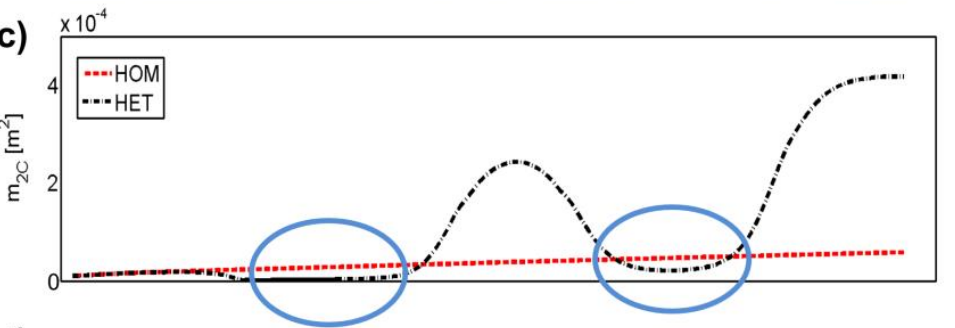

(d)

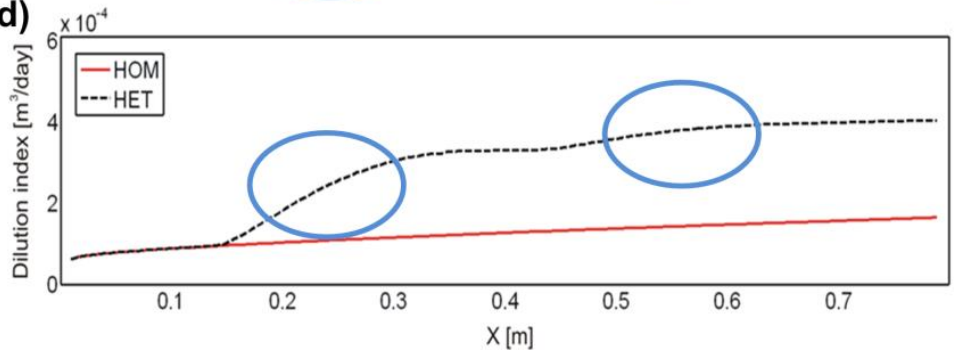

Figure 9. Solute plumes in homogeneous (a) and heterogeneous (b) laboratory scale setups, and computed second central moments (c) and flux-related dilution index (d) along the flow direction (modified after Rolle et al. 2009).

The computed second central moments of the solute distribution in the lateral direction cannot capture the enhancement of mixing due to flow focusing and show lower values in the highpermeability inclusions compared to the homogeneous setup. The flux-related dilution index, instead, allows the correct quantification of mixing in the two setups and adequately captures the monotonic increase of plume dilution and the enhancement due to flow focusing. In the 
homogeneous medium $E_{Q}(x)$ shows a slow increase throughout the length of the experimental setup. In the heterogeneous system the flux-related dilution index is the same in the low permeability matrix (same material as in the homogeneous setup) but suddenly increases as the plume is focused in the first high-permeability inclusion. Here, although the plume is less spread, mixing is significantly enhanced. The trend of $E_{Q}(x)$ is monotonically increasing also in the heterogeneous system, but the rate of increase is remarkably different at different spatial locations. For instance, the second high-permeability zone yields an increase of mixing compared to the matrix, but such increase is lower than in the first inclusion since the plume entering the downgradient high-K zone is already more well-mixed with the surrounding clean water.

The flux-related dilution index can be applied to quantify mixing at different scales. Figure 10 shows results of simulations performed at pore and field scales. The continuously released plumes in the two setups are illustrated in Figure 2 and the simulations were performed considering compounds with different aqueous diffusion coefficients spanning the typical range of variability for aqueous diffusivity in groundwater systems. The field scale simulations were performed in a stochastically generated permeability field with statistical properties consistent with the observations at the Columbus (MADE) field site (e.g., Rehfeld et al. 1992; Zheng et al. 2011). The results show the increase of plume dilution with travel distance both at the pore and at the field scale. The trend at the pore scale is more regular since the pore scale domain, though heterogeneous, was constructed to reproduce macroscopically homogeneous media used in laboratory flow-through experiments (Hochstetler et al. 2013). At the field scale, the pattern of $E_{Q}(x)$ is more irregular with many flow focusing and defocusing events due to the high permeability contrast and heterogeneity. An important outcome of this multiscale analysis is the importance of aqueous diffusion for plume dilution at different scales and the capability of the proposed metric of mixing to capture this effect. Although diffusive processes occur at the small scale and the magnitude of aqueous diffusion coefficient may be perceived as small, their importance is pivotal for mixing in porous media and their effect propagate through scales and remain important for solute transport also at large spatial scales typical of field applications.

(a) Pore Scale

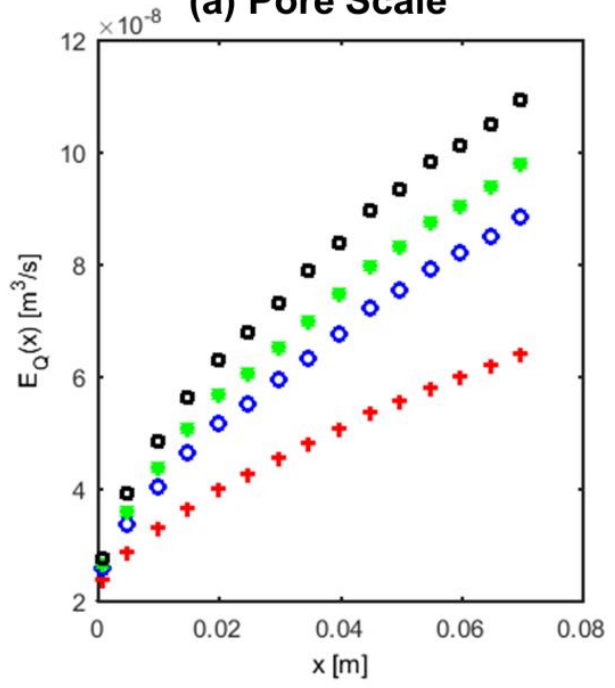

(b) Field Scale

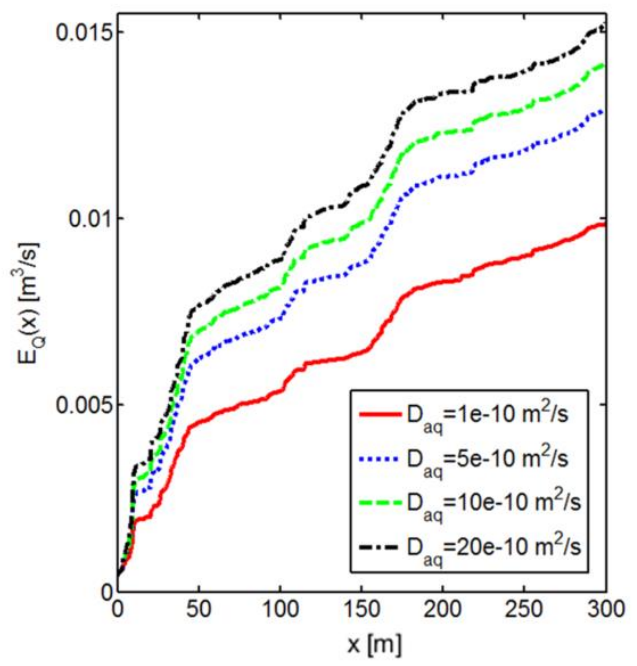

Figure 10. Flux-related dilution index for continuously injected plumes: simulations illustrating the impact of diffusion on plume dilution at the pore scale (a) and at the field scale (b) (modified after Rolle et al. 2013a). 


\section{Mixing dynamics and reactive processes}

Mixing dynamics play a crucial role for triggering and sustaining many biogeochemical reactions in porous media (Valocchi et al. 2018). A number of relevant mixing-controlled reactive systems have been studied using both experimental and modeling approaches. Probably the most studied reactive system in the context of mixing-controlled reactive transport in porous media is the reaction between two dissolved reactants resulting in a dissolved product according to: $\mathrm{A}+\mathrm{B} \rightarrow$ $\mathrm{C}$, and with kinetics described as an instantaneous or a bimolecular reaction rate (e.g., Cirpka and Valocchi 2007; Willingham et al. 2008). De Simoni et al. (2005 and 2007) proposed approaches to solve mixing-controlled reactive transport problems involving multispecies transport and heterogeneous reactions with mineral precipitation and dissolution. Mixing processes were found to control equilibrium reaction rates, which were proportional to the rates of mixing. Therefore reactions can be induced by simply mixing waters under different equilibrium conditions as can happen, for instance, in carbonate systems. In analogy to the study of mixing for conservative solutes discussed above, in the following we provide an overview of the reactive lamella theory and the quantification of mixing with the dilution index in case of reactive transport.

Reactive lamella theory. In mixing fronts involving initially segregated reactants, reaction rates are enhanced by front deformation (de Anna et al. 2014a, Le Borgne et al. 2014). Using the lamella theory, equation 5 may be used to quantify the effect of deformation on effective reaction rates. We present below the analytical expressions derived for the case of an irreversible reaction at arbitrary Damköhler numbers (Bandopadhyay et al. 2017) and that of a fast reversible reaction triggered by mixing of fluids at different chemical equilibrium (Le Borgne et al. 2014). The effective reaction rate is defined as:

$$
R=\frac{d m_{c}}{d t}
$$

where $m_{c}$ is the total mass of product generated in the front.

We first consider the case of an irreversible reaction, involving two reactants on each side of the front, $\mathrm{A}$ and $\mathrm{B}$ that react to give a product $\mathrm{C}$ :

$$
\mathrm{A}+\mathrm{B} \rightarrow \mathrm{C}
$$

with the local reaction rate:

$$
\frac{d C_{A}}{d t}=-k C_{A} C_{B}
$$

The effective reaction rates for a segment of the front of elongation subjected to a simple shear flow, $\rho=1+\nabla v t$ (Fig. 3a), are summarized in Table 2. For times smaller than the characteristic shear time $\nabla v^{-1}$, the elongation $\rho$ is close to one and the reaction rate follows the effective kinetics expected for simple diffusion fronts (Larralde, 1992): for times smaller than the characteristic reaction time $\tau_{r}=1 / k A_{0}$, the reaction rate increases as $R \sim t^{1 / 2}$, while for times larger than $\tau_{r}$ it decays as $R \sim t^{-1 / 2}$. This behavior is significantly modified by shear for $\mathrm{t}>\nabla v^{-1}$, and the reaction rates scale as $R \sim t^{3 / 2}$ for $t<\tau_{r}$ and $R \sim t^{1 / 2}$ for $t>\tau_{r}$. These findings show that the effective kinetics of reaction fronts result from a close coupling between front deformation, diffusion and local reaction kinetics. This approach may be generalized to heterogeneous porous structures and permeability fields for which the estimated reaction kinetics differ significantly from those measured in well mixed batch reactors (Dentz et al. 2011). 
Table 2. Synthesis of effective reaction rates for a mixing front subjected to a shear flow in the case of an irreversible reaction, where the Peclet number is $P e=\nabla v \tau_{D}$, the Damköhler number is $D a=\tau_{D} / \tau_{r}$, the characteristic diffusion time is $\tau_{D}=s_{0}^{2} / D$, and the characteristic reaction time is $\tau_{r}=1 / k A_{0}$

\begin{tabular}{cccc}
\hline $\begin{array}{c}\text { weak stretching } \\
\text { Pe }<\mathrm{Da}\end{array}$ & $\boldsymbol{t}<\boldsymbol{\tau}_{\boldsymbol{r}}$ & $\boldsymbol{\tau}_{\boldsymbol{r}}<\boldsymbol{t}<\boldsymbol{\nabla} \boldsymbol{v}^{-\mathbf{1}}$ & $\boldsymbol{\nabla} \boldsymbol{v}^{\mathbf{1}}<\boldsymbol{t}$ \\
& $R \sim D a\left(t / \tau_{D}\right)^{1 / 2}$ & $R \sim t^{-1 / 2}$ & $R \sim P e\left(t / \tau_{D}\right)^{1 / 2}$ \\
\hline $\begin{array}{c}\text { strong stretching } \\
\mathrm{Pe}>\mathrm{Da}\end{array}$ & $\boldsymbol{t}<\boldsymbol{\nabla} \boldsymbol{v}^{-\mathbf{1}}$ & $\boldsymbol{\nabla} \boldsymbol{v}^{-\mathbf{1}}<\boldsymbol{t}<\boldsymbol{\tau}_{\boldsymbol{r}}$ & $\boldsymbol{\tau}_{\boldsymbol{r}}<\boldsymbol{t}$ \\
$R \sim D a\left(t / \tau_{D}\right)^{1 / 2}$ & $R \sim P e D a\left(t / \tau_{D}\right)^{3 / 2}$ & $R \sim P e\left(t / \tau_{D}\right)^{1 / 2}$ \\
\hline
\end{tabular}

We now consider a reactive front involving a fast reversible reaction:

$$
\mathrm{A}+\mathrm{B} \leftrightarrow \mathrm{C}
$$

where $\mathrm{C}$ is a precipitate and the concentration of $\mathrm{A}$ and $\mathrm{B}$ are given by the mass action law (approximating the activities of the dissolved species by their concentrations):

$$
K=C_{A} C_{B}
$$

Each side of the front contains different concentrations of compounds A and B. Both fluids are at equilibrium with $\mathrm{C}$, hence the concentrations of $\mathrm{A}$ and $\mathrm{B}$ follow equation 17, although they are different on each side of the front. In this situation, mixing induces disequilibrium, which is balanced by a fast reaction in the front. While for the simple reaction given by equation 16 , this reaction is a precipitation, different chemical systems under similar hypotheses can lead to dissolution (de Simoni et al. 2007, Hidalgo et al. 2015). This framework has been used to model reactive mixing processes in freshwater/saline water fronts (Pool and Dentz 2017) and in $\mathrm{CO}_{2}$ sequestration processes (Hidalgo et al. 2015).

For the chemical system defined by equations 16 and 17, the local reaction rate in the mixing front, assuming same diffusive/dispersive properties of the reactants, can be obtained considering the conservative component:

$$
u=C_{A}-C_{B}
$$

and reads as (de Simoni et al. 2005, Le Borgne et al. 2014) :

$$
r=\frac{d^{2} C_{i}}{d u^{2}} D(\nabla u)^{2}
$$

The first term of equation 19 is linked to the chemical properties and is deduced from the relation:

$$
C_{A}=\frac{u}{2}+\sqrt{\frac{u^{2}}{4}+K}
$$

which results from solving the system of equations given by 17 and 18 . The second term of equation 19 is the scalar dissipation rate (equation 9), which quantifies the effect of mixing on reactions.

Expressions for the effective reaction rate derived from equation 19 are detailed in Le Borgne et al. (2014) for different front deformation dynamics. They are similar to those derived for the scalar dissipation rate (equation 10 and Figure 8). Following this approach, the effect of front 
deformation on the enhancement of effective reaction rates can be derived for fast equilibrium reactions.

Continuously injected reactive plumes. Interesting insights on the interplay between mixing dynamics and reactive processes are provided by entropy balances that allow the connection of the concept of the dilution index with reactive mixing (Chiogna et al. 2012; Chiogna and Rolle 2017; Ye et al. 2018). Focusing on steady-state transport and continuous release of dissolved plumes, Chiogna et al. (2012) derived the transport equation for the entropy density of a reactive species:

$$
\mathbf{v} \cdot \nabla\left(-p_{Q} \ln p_{Q}\right)-\nabla \cdot\left(\mathbf{D} \nabla\left(-p_{Q} \ln p_{Q}\right)\right)=-\underbrace{\left(1+\ln p_{Q}\right) r_{i}^{*}}_{\text {reactive mixing term }}+\underbrace{\frac{1}{p_{Q}} \nabla p_{Q}{ }^{T} \mathbf{D} \nabla p_{Q}}_{\text {dilution term }}
$$

The entropy balance for a reactant A (i.e., reactive species undergoing a homogeneous irreversible reaction as in Eqn. 14) involves two source/sink terms. The dilution term is a positive contribution and is the only term in the entropy balance for a conservative solute. The additional term is the reactive mixing contribution that acts as the only possible sink for the entropy. The interplay between these terms determines the mechanisms controlling transport and reactive mixing of a reactive species. For instance, Figure 11 illustrates the behavior for transport in heterogeneous porous media. Conservative and reactive plumes are continuously injected in a 2-D heterogeneous domain with statistics consistent with the Columbus aquifer (Rehfeldt et al. 1992). A conservative plume is shown in Figure 11a. Two scenarios were considered for reactive transport: (i) an instantaneous complete bimolecular reaction between the reactants A and B, and (ii) a slow degradation kinetics represented with a double Monod formulation dependent on the concentrations of the two reactants. The computed flux-related dilution index for the conservative and two reactive scenarios is shown in Figure 11b. In the conservative case the flux-related dilution index shows a monotonic increase consistent with the only presence of a source term in the entropy balance (Eqn. 21). Steeper increases of the flux-related dilution index correspond to zones of the domain where dilution is enhanced by flow focusing. The entropy of the reactive tracer, $E_{Q}(A)$, undergoing degradation with instantaneous and slow degradation rates is lower than the conservative case (Figure 11b). However, for the first zone of the domain (i.e., gray area in Figure 11) the trends for the two reactive cases is also increasing and the spatial derivatives have similar patterns and are positive. In this zone the plumes of reactant A are mainly diluted as the dilution terms dominate the entropy balance. Farther downgradient, the trends differentiate remarkably as the importance of the reactive mixing terms increases. In the case of instantaneous reaction, the entropy of the reactant's plume decreases showing that the reactive term has become dominant in the entropy balance. In the case of slow double Monod kinetics, dilution is the dominant mechanism throughout the domain since lateral mixing distributes the reactant within the water flux more effectively than the mass-removal caused by the reaction. Nonetheless, the degradation of the reactant at the plume fringes causes significant difference in plume dilution with respect to the conservative case. This example is illustrative of the insights on the interplay between mixing and reactive processes provided by studying the entropy of solute plumes. 

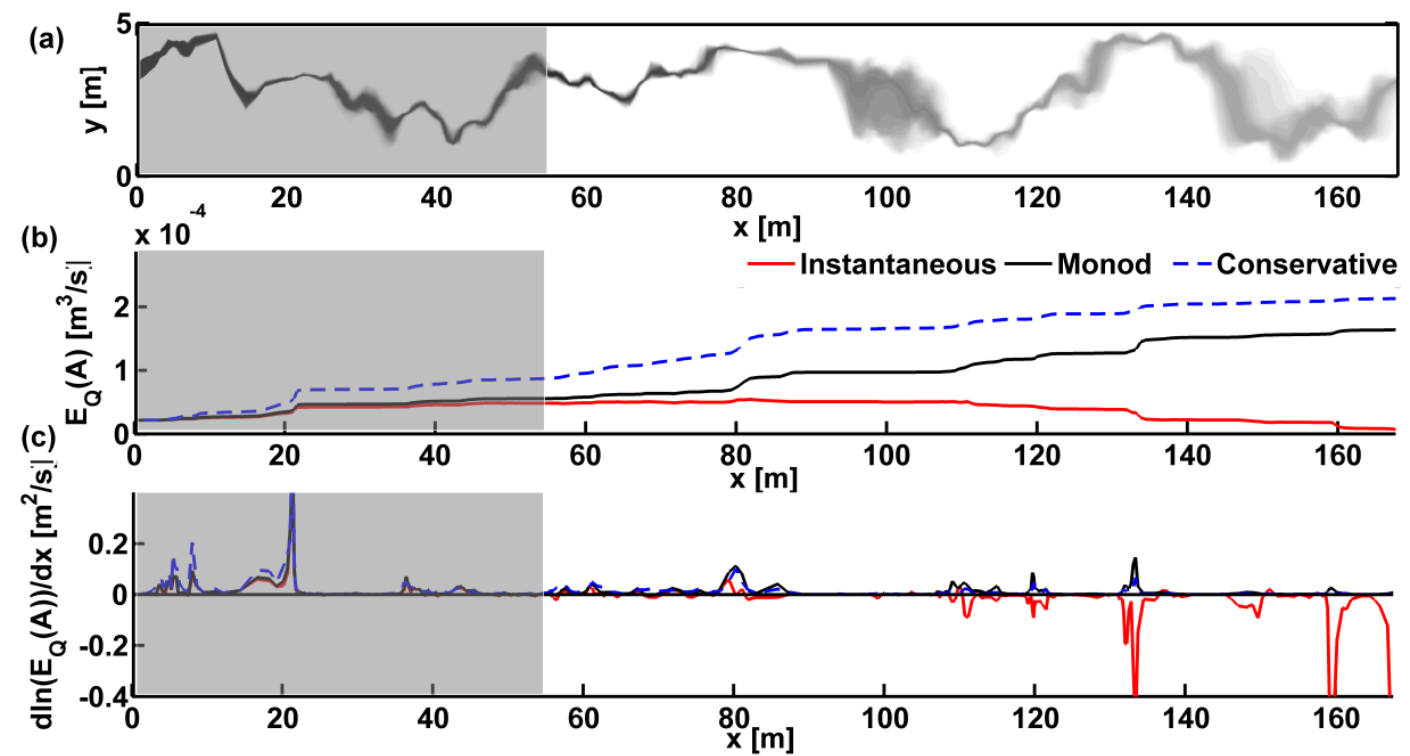

Figure 11. Entropy balance and field-scale dilution of a reactant: (a) conservative plume in a heterogeneous domain; (b) flux-related dilution index of solute A for the conservative and reactive scenarios; (c) spatial derivative of its natural logarithm (from Chiogna et al. 2012).

\section{EXAMPLES OF MIXING AND REACTIONS AT DIFFERENT SCALES}

In this chapter, we discuss a few selected examples of subsurface mixing and reaction processes at different scales. These examples cover the relevant scales of subsurface investigation, from pore to field scale, and are typically based on detailed experimental observations coupled with modelbased interpretation.

\section{Micromodel experiments}

Micromodels and microfluidic experiments have been increasingly used for the detailed investigation of transport processes and reactions in porous media (e.g., Chomsurin and Werth 2006; Zhang et al. 2010a and 2010b). Here we briefly illustrate two selected examples of studies that investigated reactive transport at the pore scale with focus on a homogeneous reaction (de Anna et al. 2014b) and on a heterogeneous reaction (Zhang et al. 2010b), respectively.

Chemiluminescence. To visualize and quantify the effect of mixing on chemical reactions at the pore scale, de Anna et al. (2014b) designed a micromodel experiment based on chemiluminescence (Fig. 12). In this reaction, photons are produced when two compounds A and $\mathrm{B}$ are mixed. In a two-dimensional micromodel composed of pillars, the light intensity quantifies the distribution of reaction rates in a mixing front, where A displaces B. The reactive fronts is composed of elongated filaments of high light intensity, i.e. high reaction rates, created by the effect of stretching due to velocity gradients at pore scale. As discussed above, this deformation process increases the area available for diffusive mass transfer and enhances concentration gradients. This increases the effective reaction rate, quantified here from the global light intensity produced in the mixing front (Fig. 12). 
(a)

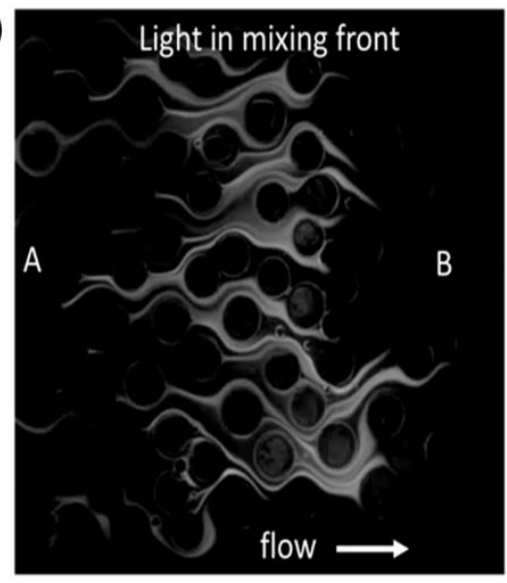

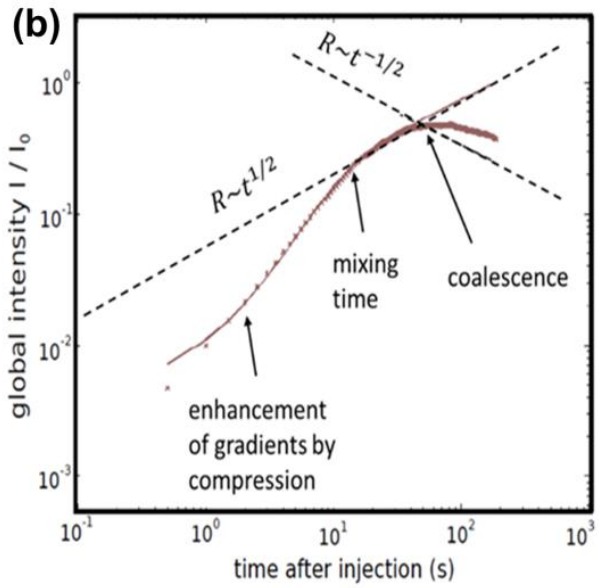

Figure 12. (a) Image of a light intensity in a $2 \mathrm{D}$ micromodel, where a compound A displaces a compound B and light is produced at the mixing front from a chemiluminescent reaction; (b) temporal evolution of the global intensity of light for different flow rates, adapted from de Anna et al. (2014b).

For a micromodel composed of random cylindrical pillars, stretching is approximately linear, since it is dominated by the shear induced by the velocity gradient between the no-slip boundary condition at the grain walls and the maximum velocity at the pore center (Table 1). The scaling that is expected for shear-enhanced mixing at large Damköhler number is $R \sim \mathrm{t}^{1 / 2}$ (Table 2). This behavior is observed in this investigation, but only for part of the experiment (Fig. 12b). Two important phenomena change this scaling. Since chemical gradients were not initially sharp in the experiment, the initial compression of the plume enhances these gradients, which accelerates the reaction compared to the simple $\mathrm{R} \sim \mathrm{t}^{1 / 2}$ scaling. This behavior is observed until the mixing time (Table 1), when diffusion overcomes compression and concentration gradients are no longer enhanced. At later time, folding of the plume over itself promotes the coalescence of filaments. Hence, in this regime, the length of the area available to diffusive mass transfer ceases to increase. This leads to a $\mathrm{R} \sim \mathrm{t}^{-1 / 2}$ scaling, which corresponds to a diffusing front in the absence of shear (Larralde et al. 1992). In this late time regime, the effect of incomplete mixing tends to be negligible and the effective reaction rate may be evaluated by conventional dispersion theory. These observations hence provide a direct visualization and a quantitative measurement of the effect of the front deformation on the reaction kinetics at pore scale.

Calcite precipitation. Mixing induced mineral precipitation and dissolution have important implications for many subsurface applications including geological $\mathrm{CO}_{2}$ sequestration, groundwater contamination and remediation, nuclear waste storage, and enhanced oil recovery. Here we briefly outline the study on mineral precipitation performed by Zhang et al. (2010b), where they carried out an experimental investigation of calcium carbonate precipitation induced by transverse mixing. Microfluidic experiments were performed in a micromodel pore structure in which solutions of calcium chloride and sodium carbonate at different saturation states where injected from two separate inlet channels (Fig. 13). Transverse mixing along the micromodel centerline allowed the contact between the reactants and the precipitation of $\mathrm{CaCO}_{3}$. Calcium carbonate precipitates were observed primarily in the central mixing zone in all the experiments performed at different saturation states. Mineral characterization showed that vaterite and calcite were the predominant precipitate forms. Zhang et al. (2010b) observed that the amount of calcium carbonate precipitation decreased with increasing saturation state, showing that denser precipitates with lower porosity were formed at higher saturation. Such precipitates blocked the pore space and reduced the transverse mixing between calcium and carbonate. The reduction in porosity and mixing can lead to a reduction of the permeability of porous media and can limit mineral 
transformation towards more stable phases. This can have important consequences in flow-through systems where mixing of fluids is coupled with mineral precipitation reactions and can affect the performance of subsurface interventions causing, for instance, pressure buildup, decreased

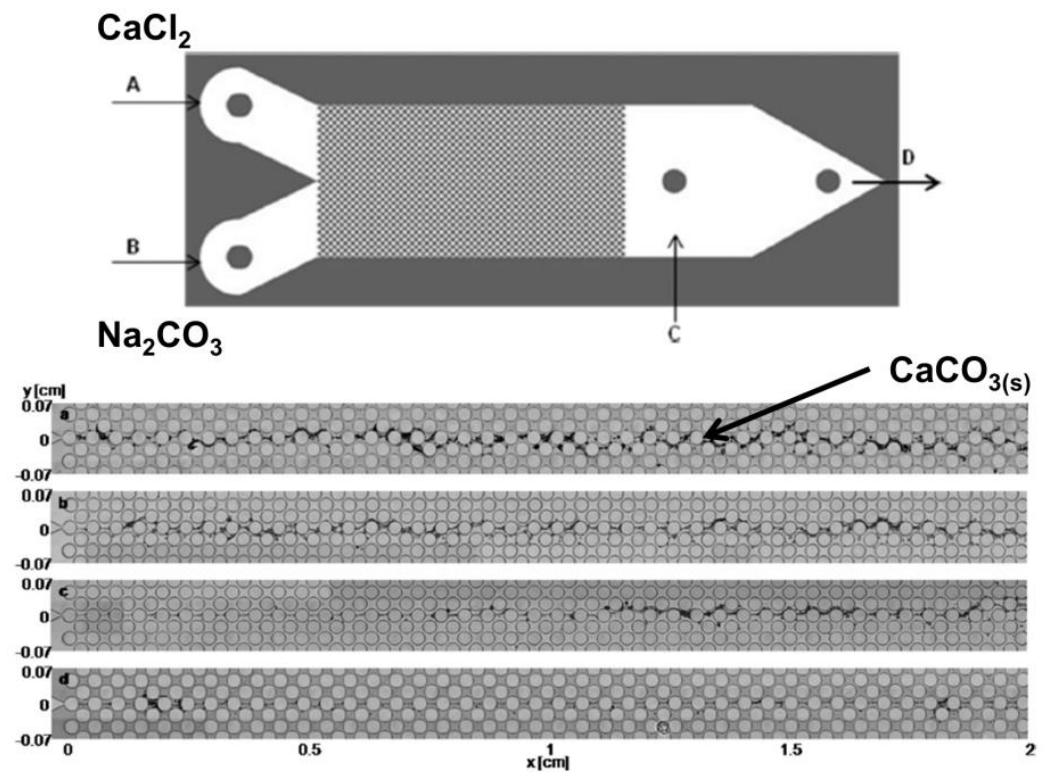

injectivity of fluids, and reduced remediation efficiency.

Figure 13. Micromodel setup and images of $\mathrm{CaCO}_{3}$ precipitates formed along the mixing zone at different saturation states (from Zhang et al. 2010b).

\section{Intermediate laboratory scale experiments}

Multidimensional laboratory flow-through experiments are instrumental for the investigation of mixing and mixing-controlled reactions in porous media. Different reactive systems have been investigated to study the interactions between mixing and reaction dynamics in porous media, including abiotic reactions between dissolved reactants (e.g., Gramling et al. 2004; Katz et al. 2010; Rolle et al. 2009), transport of pH fronts and electrostatic interactions (e.g., LoyauxLawniczak et al. 2012; Muniruzzaman et al. 2014; Muniruzzaman and Rolle 2015), precipitation/dissolution reactions (e.g., Tartakovsky et al. 2008; Poonoosami et al. 2015; Haberer et al. 2015; Battistel et al. 2019), and microbially-mediated reactions (e.g., Thullner al. 2002; Bauer et al. 2009a; Song et al. 2014). In the following, we provide two examples of mixing investigation in flow-through laboratory setups considering abiotic and biotic mixing-controlled reactions.

Abiotic mixing-controlled reactions. Reactive mixing and mixing enhancement by flow focusing and by transient flow fields was investigated by Rolle et al. (2009). Flow-through experiments were performed in a quasi two-dimensional flow-through chamber with dimensions: $77.9 \mathrm{~cm} \times 15.0 \mathrm{~cm} \times 1.1 \mathrm{~cm}$. The setup was filled with a homogeneous porous medium with a grain size of $0.25-0.3 \mathrm{~mm}$; high-permeability inclusions were embedded to realize a heterogeneous setup by using a coarser grain size $(1-1.5 \mathrm{~mm})$. The considered reactive system was an acid/base reaction between an alkaline $(\mathrm{NaOH})$ and an acidic $(\mathrm{HCl})$ solution. Such reaction occurs rapidly, thus, it is suitable to investigate reactive mixing in porous media. The alkaline solution $(\mathrm{pH}=11.49)$ was continuously injected from the two central inlet ports of the flow-through chamber, whereas the acidic solution $(\mathrm{pH}=2.03)$ was injected from the surrounding inlet ports. The solutions contained bromophenol blue as $\mathrm{pH}$ indicator that allowed the visualization of the alkaline plume 
(Fig. 14). The acid/based reaction occurring at the plume fringes resulted in a steady-state alkaline plume within the porous media. In the homogeneous setup the plume had a length more than half the length of the flow-through chamber (Fig. 14a). The shape of the plume and its length were found to be considerably different in the experiments performed in the heterogeneous system. Here the alkaline plume disappeared at the location of the first high-permeability inclusion where mixing is enhanced by focusing of flow within the thin zone of coarser permeable material. Since the reaction was fast the enhancement of mixing directly translated into a reaction enhancement leading to a remarkably shorter steady-state plume. Considerably higher concentrations of $\mathrm{NaOH}$ in the same $\mathrm{HCl}$ background were necessary to establish longer plumes in the heterogeneous porous medium. A more than 7-fold increase of the $\mathrm{NaOH}$ concentration was required to obtain a steady-state alkaline plume reaching the outlet of the flow-through setup.

Experiments were also carried out to investigate the effect of transient flow conditions on reactive mixing. An oscillating flow field was established by using different flow rates and by pumping from alternating inlet and outlet ports. Such flow field caused the alkaline plume to bend and oscillate within the homogeneous porous medium (Fig. 14c). The experimental results showed transient alkaline plumes with lengths comparable to the one observed in the same porous medium under steady-state conditions.

Numerical simulations allowed the quantitative comparison between reactive mixing enhancement by flow focusing and transient flow. The results showed that both mechanisms contributed to increase reactive mixing. However the magnitude of reactive mixing enhancement was found to be considerably larger when the plume was focused in high-permeability inclusions. In fact, whereas the oscillating flow only caused moderate local mixing enhancement the focusing of the alkaline plume in the high-K inclusion resulted in a remarkable increase of reactive mixing.

Figure 14. Abiotic mixing-controlled reactions: experimental observations and simulated results (contour at $\mathrm{pH}=4.6$ ).
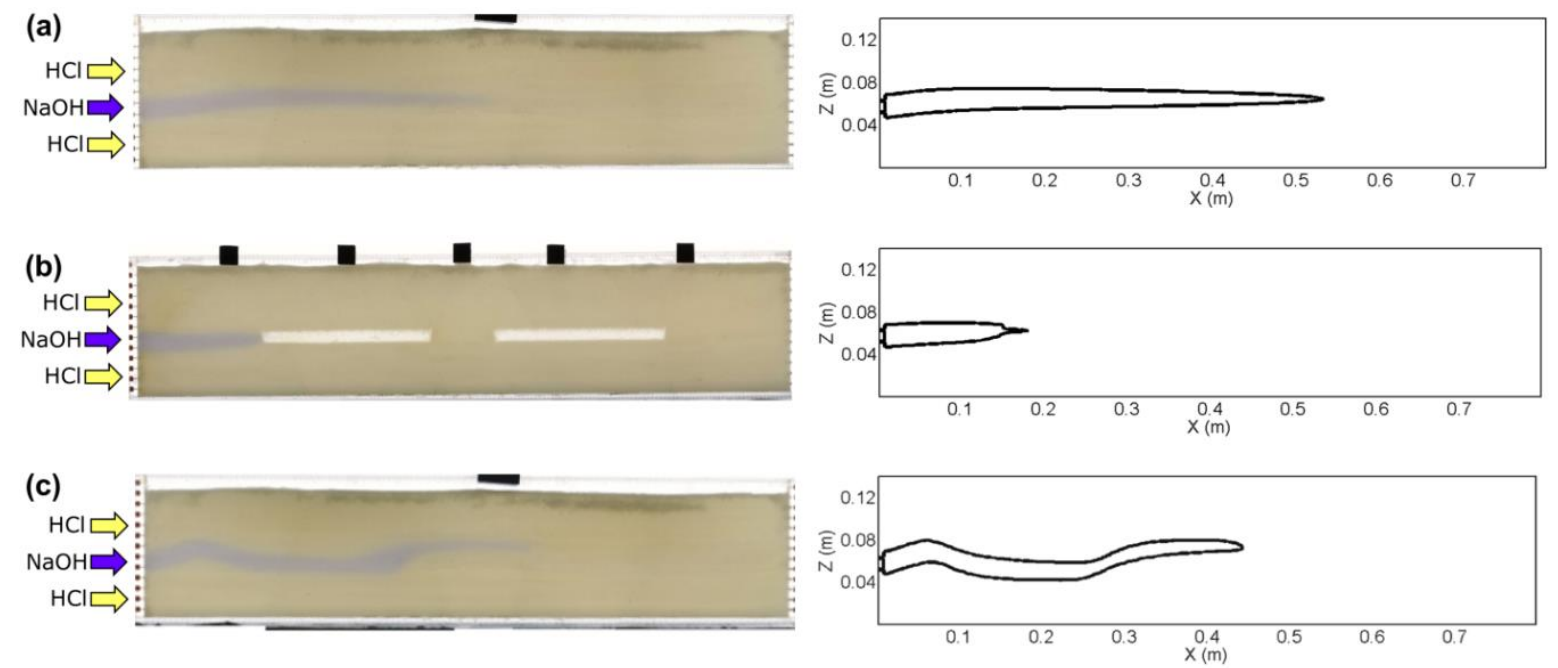

Steady-state plume in the homogeneous medium (a), steady-state plume in the heterogeneous medium (b), and plume under transient conditions in the homogeneous setup (c) (modified after Rolle et al. 2009).

Mixing and biodegradation. The coupling of mixing processes with microbially-mediated reactions is of utmost importance in subsurface porous media. For instance, biodegradation of organic contaminants often depends on the contact between substrates (e.g., soluble electron donors and electron acceptors) and nutrients at the fringes of pollutants' plumes. Laboratory flowthrough microcosms (Bauer et al. 2009a) have been used to study biodegradation of common groundwater contaminants. Here we briefly illustrate some of the findings by Bauer et al. (2008) who performed flow-through biodegradation experiments to investigate microbially-mediated 
degradation of petroleum hydrocarbons under flow-through conditions. The setup was a quasi 2D flow-through chamber similar to the one described above. A dissolved toluene plume was continuously injected from a central inlet whereas growth medium containing nutrients and electron acceptors was injected from the surrounding inlet ports. Experiments were performed under aerobic and anaerobic conditions. Two pure strains were considered: Pseudomonas putida mt-2 capable of aerobic degradation of toluene, and the Aromatoleum aromaticum strain EbN1 that degrades toluene using nitrate as electron acceptor. The degradation reactions under aerobic and anaerobic conditions can be written as:

$$
\begin{gathered}
\mathrm{C}_{7} \mathrm{H}_{8}+9 \mathrm{O}_{2}+3 \mathrm{H}_{2} \mathrm{O} \rightarrow 7 \mathrm{HCO}_{3}^{-}+7 \mathrm{H}^{+} \\
\mathrm{C}_{7} \mathrm{H}_{8}+0.2 \mathrm{H}^{+}+7.2 \mathrm{NO}_{3}^{-} \rightarrow 7 \mathrm{HCO}_{3}^{-}+3.6 \mathrm{~N}_{2}+0.6 \mathrm{H}_{2} \mathrm{O}
\end{gathered}
$$

Biodegradation takes place when the main reactants (i.e., the organic contaminant and the electron acceptors) come into contact. This occurs in thin ( $\mathrm{mm}$ to $\mathrm{cm}$ scale) fringes surrounding the contaminant plumes where transverse dispersion allows the reactants to mix (Fig. 15). Such fringe zones represent biodegradation hotspots and are of fundamental importance to understand the transport and fate of contaminants in the subsurface. At the plume fringes reactants show steep concentration gradients that are the driving force for lateral mixing. The contact between initially segregated reactants (i.e., the organic contaminant in the plume and the electron acceptors in the background groundwater solution) provides optimal conditions for the biomass to grow. Highresolution subcoring of the porous medium and successive cell counts for the different samples allowed resolving the vertical distribution of the degrading biomass. The results show that highest numbers of degrading bacteria were found at the plume fringes (Fig. 15).

Successive studies investigated biodegradation of different monoaromatic petroleum hydrocarbons with focus on the effects of hydraulic heterogeneities on contaminant biodegradation (Bauer et al. 2009b) and on isotope fractionation of organic pollutants during fringe-controlled biodegradation (Rolle et al. 2010). Flow-through microcosms and their model-based interpretation have provided important insights on the coupling of biodegradation reactions with physical transport and mixing processes in porous media.

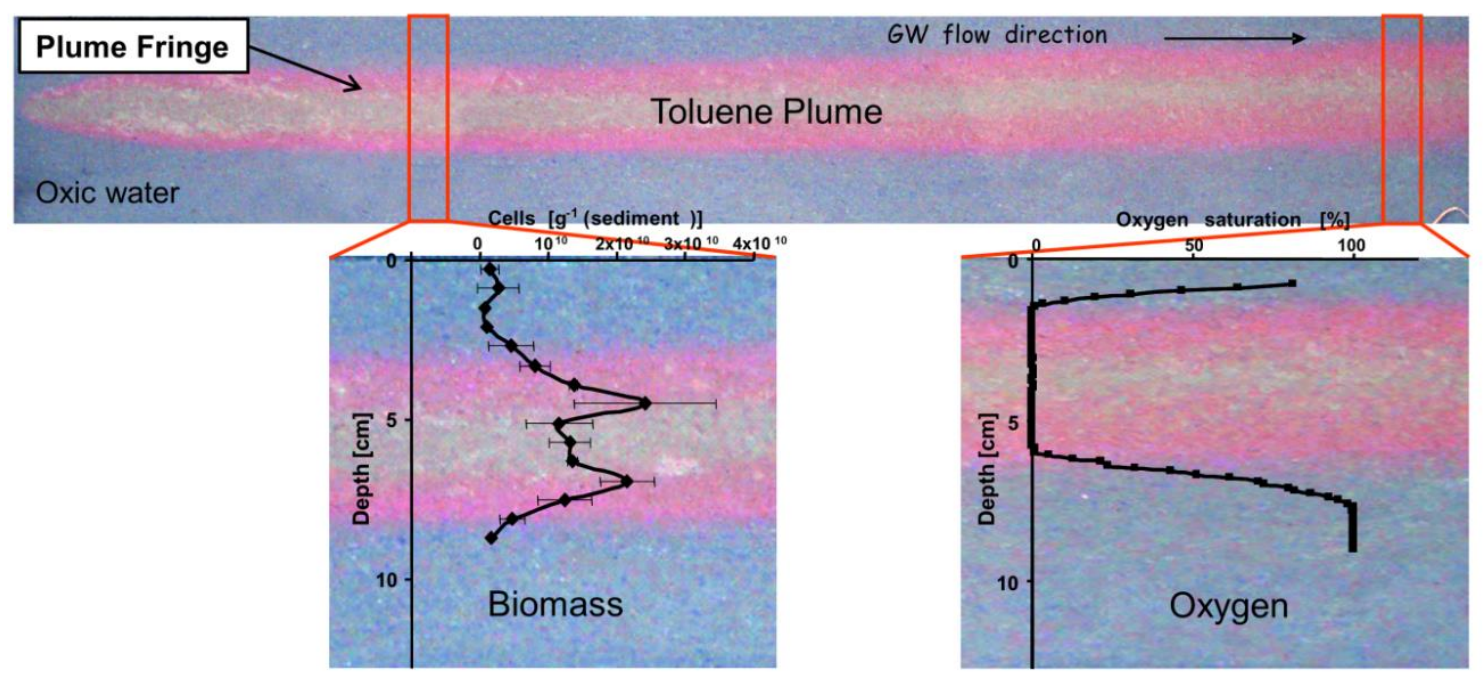

Figure 15. Toluene plume with bioactive fringes visualized with a redox indicator and results showing enhanced biomass growth and steep oxygen gradients at the plume fringes (modified after Bauer 2007 and Bauer et al. 2008).

\section{Field-scale investigation}


The investigation of mixing processes at the field scale is very challenging due to the heterogeneity of geologic media and to the complex interplay of physical, chemical, and biological processes that control mixing and reactive transport in the subsurface. Here we present two examples of investigation of mixing processes relevant for the fate of important organic and inorganic contaminants.

Biogeochemical gradients in a contaminated aquifer. The release of organic compounds in the subsurface triggers important redox processes that can results in a sequence of terminal electron acceptor processes and in a typical redox zonation of the contaminated aquifer (Chapelle et al. 1995; Christensen et al. 2001; Rolle et al. 2008). The redox processes can involve dissolved species and occur at the fringes of contaminant plumes, or solid electron acceptors such as reactive minerals (e.g., iron and manganese oxides and hydroxides) and, thus, take place in the core of the plume. Important electron acceptors that are replenished with the clean groundwater and come into contact with the dissolved organic contaminant plume through transverse mixing include dissolved oxygen, nitrate and sulfate. Plume fringes are typically very thin also at the field scale due to the limited extent of transverse mixing. To characterize these bioactive zones depth-specific and highresolution sampling is required (e.g., Tuxen et al. 2006; Anneser et al. 2008; Rasa et al. 2011). Anneser et al. 2008 performed a detailed investigation of biogeochemical gradients in a tar-oil contaminated aquifer. The site was a former gasworks facility where the shallow Quaternary sand and gravel aquifer was contaminated by monoaromatic and polycyclic aromatic hydrocarbons. Dissolved plumes originated from the source zone and were transported downgradient. The main plume had a length of $200 \mathrm{~m}$ and was very shallow $(\sim 1 \mathrm{~m})$. The groundwater geochemistry was considerably altered by the release of the organic contaminants and resulted in distinct biogeochemical gradients. A special multilevel monitoring well with very high spatial resolution (up to $2.5 \mathrm{~cm}$ ) was installed with the aim of resolving the steep biogeochemical gradients at the site (Fig. 16).

(a)

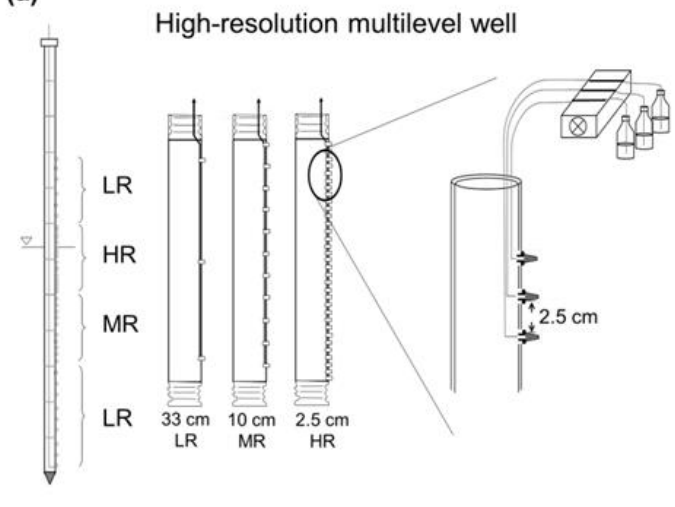

(b)

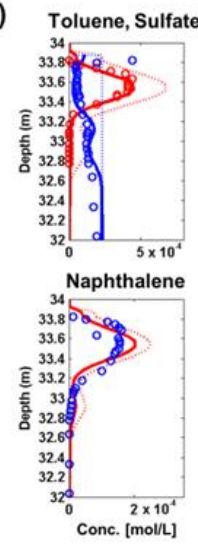

(c)
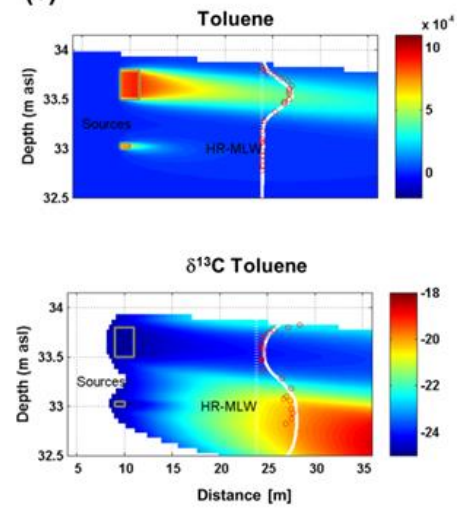

Figure 16. Plume fringes and biogeochemical gradients in a contaminated aquifer: (a) high-resolution multilevel well, (b) measured and simulated concentrations of two contaminants and of the main electron acceptor, (c) 2-D simulations and measurements of toluene concentrations and carbon isotopes (modified after Anneser et al. 2008 and Prommer et al. 2009).

The high-resolution sampling revealed very pronounced variation with depth of the contaminants' concentrations, groundwater species, isotopic signatures, and general parameters such as $\mathrm{pH}$ and redox potential. The results of the high-resolution investigation of the biogeochemical active plume fringes at the site were compared with a conventional multi-level well with spatial resolution of 0.5-1 $\mathrm{m}$. The high-resolution investigation revealed $\mathrm{cm}$-scale gradients of contaminants, electron acceptors, and metabolites that could not be resolved with the 
conventional multilevel sampling.

At the same field site, Winderl et al. (2008) used the sediment cores from the installed multilevel well to carry out a fine-scale study of the microbial community in the contaminated aquifer. Different microbial techniques allowed the qualitative and quantitative characterization of aquifer microorganisms with depth. In particular, the results showed the presence of a highly specialized community of anaerobic degraders at the lower fringe of the contaminant plume within the sulfidogenic gradient zone. This investigation established a clear link between the distribution of aquifer microbial community and biogeochemical gradients and confirmed that such biogeochemical gradients are hot spots of anaerobic toluene degradation. In a follow up investigation, Prommer et al. (2009) performed a reactive transport modeling study using the highresolution dataset available at the field site. The model included flow, transport, contaminant degradation, microbial dynamics, and geochemical reactions in a two-dimensional transect along the flow direction. The simulation outcomes were compared with the high-resolution biogeochemical gradients measured in the aquifer (Fig. 16). Special focus of the model-based interpretation was the analysis of the isotopic signatures (carbon and sulfur isotopes). In particular for toluene, the most degradable compound in the plume, the simulations captured the evolution of the isotopic signature with a small enrichment in the plume core and a strong enrichment (up to $3.3 \%$ ) in the bioactive, sulfidogenic lower fringe of the plume. This finding provided additional evidence of enhanced biodegradation activity in the mixing zone between the organic contaminant plume and the surrounding groundwater rich of sulfate, the main electron acceptor at the site.

Mixing fronts and arsenic attenuation in the hyporheic zone. Reactive mixing is also a key process in hyporheic zones, which represent hot spots of mixing and reaction involving surface water and groundwater (Hester et al. 2017). As demonstrated in the study of Datta et al. (2009), when anoxic groundwater discharges in rivers, mixing with oxic water leads to oxidation of dissolved iron and precipitation of iron oxides. This precipitation process also traps other dissolved elements, such as arsenic, that can be immobilized through sorption and incorporation in iron mineral phases (Kocar et al. 2006; Jessen et al. 2012; Stolze et al. 2019). Datta et al. (2009) measured large arsenic concentrations in shallow sediments along the Meghna river in Bangladesh.

Figure 17. Arsenic concentration in shallow sediments along the Meghna river: (a) locations of sediment sampling

(a)

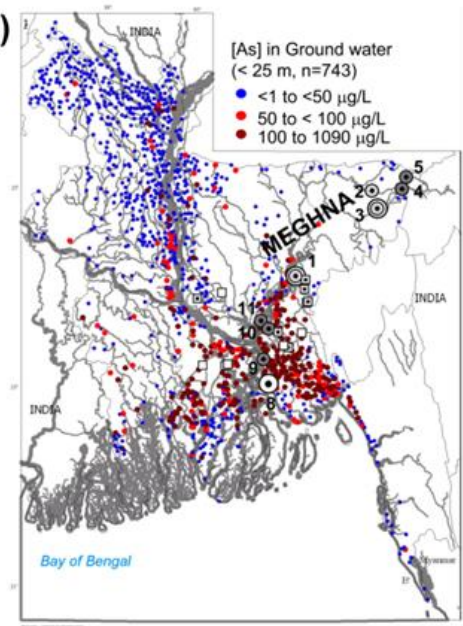

(b)

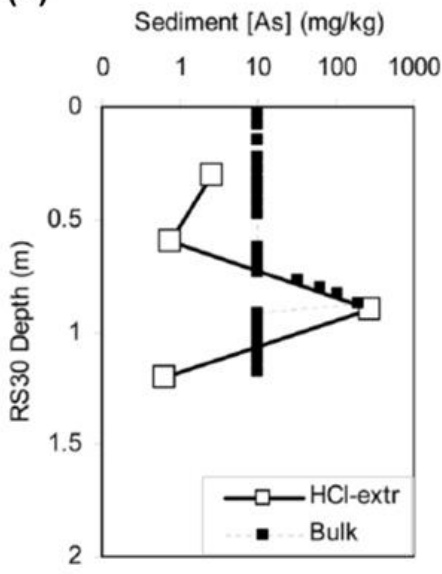

sites and groundwater As concentrations; (b) depth-profile of sediment arsenic concentrations at one of the measurement location, showing a large increase in As concentration in the mixing zone (from Datta et al. 2009).

These large concentrations were found in the redox transition zone corresponding to the mixing front between groundwater and river water, where secondary minerals where formed upon 
the contact between the anoxic and oxic waters. Enrichment in arsenic in this mixing zone led to concentrations several orders of magnitude larger than the crustal abundance. Mixing fronts at the interface between anoxic and oxic water are thus suggested to act as reactive barriers, preventing large quantities of arsenic to flow into the river. However, depending on seasonal fluctuations in water levels, the location of the redox transition zone is expected to move, which may cause reducing conditions to reestablish thus leading to the dissolution of iron oxides and to the remobilization of the trapped arsenic. Such reactive barriers may be widespread in mixing fronts between surface water (rivers, lakes, oceans) and groundwater, thus playing an important role in the cycling of elements in hydrological systems.

\section{CONCLUSIONS AND OUTLOOK}

We have provided a brief overview of some recent conceptual and experimental advances shedding new light on the temporal and spatial dynamics of mixing and reaction fronts. We have selected illustrative examples of the coupling between physical mixing processes and biogeochemical reactions in highly non-uniform subsurface systems. These examples encompass both experimental and modeling applications at different scales. Mixing fronts, whether naturally occurring in hydrological systems or resulting from contaminant plumes, are often hot spots of biogeochemical reactions, where reaction rates are orders of magnitude larger than the background. Although the investigation of mixing in subsurface systems has advanced by leaps and bounds in recent years, formidable challenges remain to be addressed in order to be able to quantify and predict mixing-controlled reactions in natural and engineered systems. In the following, we present some thoughts about investigation directions for subsurface mixing and reactive processes that, in our view, represent upcoming challenges and may attract the attention of researcher in the next years.

Mixing and reactive processes in the subsurface have been the focus of different disciplines that have addressed these problems with very different approaches and often little degree of integration. This is, perhaps, due to the sharp boundary between physics and chemistry applied to subsurface processes. For instance, the literature of subsurface hydrology has focused on heterogeneity and scales with often simplified chemistry (e.g., the typical reaction: A $+B \rightarrow C$ ) and few attempts of including realistic complex kinetic and equilibrium reaction networks. On the other hand, most studies considering the complexity of biogeochemical processes have been carried out assuming simplifying flow and transport conditions, often in homogeneous domains. We foresee a "mixing of knowledge" between physical and chemical approaches to the investigation of reactive transport processes in subsurface formations. This will lead to more integrated studies that will tackle more complex and realistic mixing-controlled reactive systems. Important reactions and interactions that have been rarely considered in the context of the study of mixing processes will likely be investigated in greater detail. Such processes include surface reactions, solid/solution interactions of organic and inorganic compounds, and charge interactions both at the surface/solution interface and within the pore water. Furthermore, advances in microbial, analytical and stable isotopes techniques will allow new possibilities for investigating microbially-mediated mixing-controlled reactions. This will lead to significant advances in understanding not only the impact of mixing processes on overall reaction rates but also on the structure and functioning of microbial communities in the subsurface (Stegen et al. 2015). Experimental and modeling studies will also be more integrated and will attempt to consider combined effects of physical and chemical heterogeneity on mixing processes in porous media.

Another major challenge is the one of dimensionality. Traditional investigations of transport processes in porous media have been largely based on column experiments. Such column investigations, and the associated one-dimensional perspective on contaminant transport, have 
many limitations and have contributed to hide the key importance of dimensionality for groundwater quality. The recent studies on mixing, some of them have been described above, have overcome such limitations and have often performed experimental and modeling investigation in 2-D setups. However, fully 3-D studies of mixing processes in porous media are still rare (e.g., Ye et al. 2015c, Rolle et al. 2018). Future experimental and modeling investigation is likely to focus on fully three-dimensional systems. The increased possibilities of high-resolution sampling and non-invasive sensor-based monitoring, as well as the advances in computational capabilities represent crucial advantages to study mixing processes in fully 3-D systems.

Issues of spatial scales and heterogeneity will still be persistent and will greatly influence the study of subsurface systems (Kitanidis 2015). Despite the great advances in describing the mechanisms of mixing and mixing-controlled reactions in porous media, the understanding and prediction of the impact of small-scale mixing processes at larger field scales remains a major theoretical and practical challenge. Furthermore, resolving coupled and dynamic mixing and reaction processes at the interface of different hydrological compartments, such as surface/subsurface environments, is an outstanding challenge, which is attracting intense research focus (e.g., Hester et al. 2017). Key challenges include: (i) understanding and quantifying the effect of temporal fluctuations in surface water dynamics on hot moments of mixing and reaction at interfaces with subsurface environments; (ii) capturing the dynamics of mixing and reaction fronts by taking advantage of technological developments, such as distributed temperature sensing, high frequency chemistry monitoring and hydrogeophysics, to resolve mixing-relevant scales at higher spatial and temporal resolution; and (iii) representing the effect of mixing hot spots and hot moments in large scale hydrological models.

Reactive transport simulators (Steefel et al. 2015) are instrumental to study mixing processes in the subsurface. Advanced multiscale modeling tools (Molins 20XX, this volume) are needed to integrate the effects of physical, chemical, biological and electrostatic interactions (e.g., Thullner et al. 2005; Appelo and Rolle 2010; Tournassat and Steefel 2015; Rasouli et al. 2015; Muniruzzaman and Rolle 2016), to quantify mixing and mixing-controlled reactions at different scales using pore, continuum and hybrid approaches (e.g., Battiato and Tartakovsky 2011; Scheibe et al. 2015), and to describe reactive transport processes across different environmental compartments and critical zones (e.g., Li et al. 2017).

\section{REFERENCES}

Acharya RC, Valocchi AJ, Werth CJ, Willingham TW (2007) Pore-scale simulation of dispersion and reaction along a transverse mixing zone in two-dimensional porous media. Water Resour Resour 43: W10435

Amos RT, Bekins BA, Delin GN, Cozzarelli IM, Blowes DW, Kirshtein JD (2011) Methane oxidation in a crude oil contaminated aquifer: Delineation of aerobic reactions at the plume fringes. J Contam Hydrol 125: 13-25

Anneser B, Einsiedl F, Meckenstock RU, Richters L, Wisotzky F, Griebler C (2008) High-resolution monitoring of biogeochemical gradients in a tar oil-contaminated aquifer. Appl Geochem 23: 1715-1730

Appelo CAJ, Rolle M (2010) PHT3D: A reactive multicomponent transport model for saturated porous media. Groundwater 48: 627-632

Babey T, de Dreuzy JR, Casenave C (2015) Multi-Rate Mass Transfer (MRMT) models for general diffusive porosity structures. Adv Water Resour 76: 146-156

Bakker M, Hemker K. (2004) Analytic solutions for groundwater whirls in box-shaped, layered anisotropic aquifers. Adv Water Res 27: 1075-1086

Bandara UC, Tartakovsky AM, Oostrom M, Palmer BJ, Grate J, Zhang C (2013) Smoothed particle hydrodynamics pore-scale simulations of unstable immiscible flow in porous media. Adv Water Resour 62: 356-369 
Bandopadhyay A, Le Borgne T, Méheust Y, Dentz, M (2017). Enhanced reaction kinetics and reactive mixing scale dynamics in mixing fronts under shear flow for arbitrary Damköhler numbers. Adv Water Resour 100: 78-95

Bandopadhyay A, Davy P, Le Borgne T. (2018) Shear flows accelerate mixing dynamics in hyporheic zones and hillslopes. Geophys Res Lett 45: 11-659

Battiato I, Tartakovsky DM (2011) Applicability regimes for macroscopic models of reactive transport in porous media. J Contam Hydrol 120-121: 18-26

Battistel M, Muniruzzaman M, Onses F, Lee J, Rolle M (2019) Reactive fronts in chemically heterogeneous porous media: experimental and modeling investigation of pyrite oxidation. Appl Geochem 100: 77-89

Bauer RD (2007) Control and limitations of microbial degradation in aromatic hydrocarbon plumes - experiments in 2-D model aquifers. PhD dissertation, University of Tuebingen, Tuebingen, Germany

Bauer RD, Maloszewski P, Zhang Y, Meckenstock RU, Griebler C (2008) Mixing-controlled aerobic and anaerobic biodegradation of toluene in porous media - results from two-dimensional laboratory experiments. J Contam Hydrol 96, 150-168.

Bauer RD, Rolle M, Kürzinger P, Grathwohl P, Meckenstock RU, Griebler C (2009a). Two-dimensional flow-through microcosms - Versatile test systems to study biodegradation processes in porous aquifers. J Hydrol 369: 284295

Bauer RD, Rolle M, Eberhardt C, Grathwohl P, Bauer S, Kolditz O, Meckenstock RU, Griebler C (2009b) Enhanced biodegradation by hydraulic heterogeneities in petroleum hydrocarbon plumes. J Contam Hydrol 105: 56-68

Bear J (1972) Dynamics of Fluids in Porous Media, Elsevier, New York

Berkowitz B, Cortis A, Dentz M, Scher H (2006), Modeling non-Fickian transport in geological formations as a continuous time random walk. Rev Geophys 44: RG2003

Bijeljic B, Muggeridge AH, Blunt MJ (2004) Pore-scale modeling of longitudinal dispersion. Water Resour Res 40: W11501

Bijeljic B, Mostaghimi P, Blunt MJ (2011) Signature of non-Fickian solute transport in complex heterogeneous porous media. Phys Rev Lett: 107, 204502

Bolster D, Dentz M, Le Borgne T (2011) Hypermixing in linear shear flow. Water Resour Res 47: W09602

Bolster D, Valdés-Parada FJ, Le Borgne T, Dentz M, Carrera J (2011) Mixing in confined stratified aquifers. J Contam Hydrol 120-121: 198-212

Bjerg PL, Tuxen N, Reitzel LA, Albrechtsen H-J, Kjeldsen P (2011) Natural attenuation processes in landfill leachate plumes at three Danish sites. Ground Water 49: 688-705

Chapelle FH, McMahon PB, Dubrovsky NM, Fujii RF, Oaksford ET, Vroblesky DA (1995) Deducing the distribution of terminal electron-accepting processes in hydrologically diverse groundwater systems. Water

Chiogna G, Rolle M (2017) Entropy-based critical reaction time for mixing-controlled reactive transport. Water Resour Res 53: 7488-7498

Chiogna G, Eberhardt C, Cirpka OA, Grathwohl P, Rolle M (2010) Evidence of compound-dependent hydrodynamic and mechanical transverse dispersion by multitracer laboratory experiments. Environ Sci Technol 44: 688-693

Chiogna G, Cirpka OA, Grathwohl P, Rolle M (2011a) Transverse mixing of conservative and reactive tracers in porous media: quantification through the concepts of flux-related and critical dilution indices. Water Resour Res 47: W02505

Chiogna G, Cirpka OA, Grathwohl P, Rolle M (2011b) Relevance of local compound-specific transverse dispersion for conservative and reactive mixing in heterogeneous porous media. Water Resour Res 47: W07540

Chiogna G, Hochstetler D.L., Bellin A, Kitanidis PK, Rolle M (2012) Mixing, entropy and reactive solute transport. Geophys Res Lett 39: L20405

Chiogna G, Rolle M, Bellin A, Cirpka OA (2014) Helicity and flow topology in three-dimensional anisotropic porous media. Adv Water Resour 73: 134-143 
Chiogna G, Cirpka OA, Rolle M, Grathwohl P (2015) Helical flow in three-dimensional nonstationary anisotropic heterogeneous porous media. Water Resour Res 51: 261-280

Chomsurin C, Werth CJ (2003) Analysis of pore-scale nonaqueous phase liquid dissolution in etched silicon pore networks. Water Resour Res 39: 1265

Christensen TH, Bjerg PL, Banwart SA, Jakobsen R, Heron C, Albrechtsen HJ (2001) Characterization of redox conditions in groundwater contaminant plumes. J Contam Hydrol 45: 165-241

Cirpka OA, Valocchi AJ (2007) Two-dimensional concentration distribution for mixing-controlled bioreactive transport in steady state. Adv Water Resour 30: 1668-1679

Cirpka OA, de Barros FPJ, Chiogna G, Rolle M, Nowak W (2011) Stochastic flux-related analysis of transverse mixing in two-dimensional heterogeneous porous media. Water Resour Res 47: W06515

Cirpka OA, Rolle M, Chiogna G, de Barros FPJ, Nowak W (2012) Stochastic evaluation of mixing-controlled steadystate plume lengths in two-dimensional heterogeneous domains. J Contam Hydrol 138-139: 22-39

Cirpka OA, Chiogna G, Rolle M, Bellin A (2015) Transverse mixing in three-dimensional nonstationary anisotropic heterogeneous porous media. Water Resour Res 51: 241-260

Crevacore E, Tosco T, Sethi R, Boccardo G, Marchisio D (2016) Recirculation zones induce non-Fickian transport in three-dimensional periodic porous media. Phys Rev E 94: 053118

Cushman JH (2013) The physics of fluids in hierarchical porous media: angstroms to miles (Vol. 10). Springer Science $\&$ Business Media.

Dagan G (1989) Flow and transport in porous formations, Springer-Verlag, Berlin

Datta S, Mailloux B, Jung H-B, Hoque MA, Stute M, Ahmed KM, Zheng Y (2009) Redox trapping of arsenic during groundwater discharge in sediments from the Meghna riverbank in Bangladesh. PNAS 106: 16930-16935.

de Anna P, Le Borgne T, Dentz M, Tartakovsky AM, Bolster D, Davy P (2013) Flow intermittency, dispersion and correlated continuous time random walks in porous media. Phys Rev Lett: 110, 184502

de Anna P, Dentz M, Tartakovsky A., Le Borgne T (2014a) The filamentary structure of mixing fronts and its control on reaction kinetics in porousmedia flows. Geophys Res Lett 41: 4586-4593

de Anna P, Jimenez-Martinez J, Tabuteau H, Turuban R, Le Borgne T, Derrien M, Méheust Y (2014b) Mixing and reaction kinetics in porous media: An experimental pore scale quantification. Environ Sci Technol 48: 508-516

de Barros FPJ, Dentz M (2016) Pictures of blockscale transport: effective versus ensemble dispersion and its uncertainty. Adv Water Resour 91: 11-22

de Barros FPJ, Dentz M, Koch J, Nowak W (2012) Flow topology and scalar mixing in spatially heterogeneous flow fields. Geophys Res Lett 39: L08404

de Dreuzy JR, Rapaport A, Babey T, Harmand J (2013) Influence of porosity structures on mixing-induced reactivity at chemical equilibrium in mobile/immobile Multi-Rate Mass Transfer (MRMT) and Multiple INteracting Continua (MINC) models. Water Resour Res 49: 8511-8530.

Delgado JMPQ (2006) A critical review of dispersion in packed beds. Heat Mass Transfer 42: 279-310

Dentz M, de Barros FPJ (2015) Mixing-scale dependent dispersion for transport in heterogeneous flows. J Fluid Mech 777: 178-195

Dentz M, Le Borgne T, Englert A, Bijeljic B (2011) Mixing, spreading and reactions in heterogeneous media: A brief review. J Contam Hydrol 120-121: 1-17

Dentz M, Lester DR, Le Borgne T, de Barros FPJ (2016) Coupled continuous-time random walks for fluid stretching in two-dimensional heterogeneous media. Phys Rev E 94: 061102

Dentz M, Icardi M, Hidalgo JJ (2018) Mechanisms of dispersion in a porous medium. J Fluid Mech 841: 851-882

De Simoni M, Carrera J, Sanchez-Vila X, Guadagnini A (2005) A procedure for the solution of multi-component reactive transport problems. Water Resour Res 41: W11410

De Simoni M, Sanchez-Vila X, Carrera J, Saaltink MW (2007) A mixing ratios-based formulation for multicomponent reactive transport. Water Resour Res 43: W07419 
Di Dato M, de Barros FPJ, Fiori A, Bellin A (2018) Improving the efficiency of 3-D hydrogeological mixers: dilution enhancement via coupled engineering-induced transient flows and spatial heterogeneity. Water Resour Res 54: 2095-2111

Druhan JL, Maher K (2017) The influence of mixing on stable isotope ratios in porous media: A revised Rayleigh model. Water Resour Res 53: 1101-1124

Eckert D, Rolle M, Cirpka OA (2012) Numerical simulation of isotope fractionation in steady-state bioreactive transport controlled by transverse mixing. J Contam Hydrol 140: 95-106

Engdahl NB, Ginn TR, Fogg GE (2013) Scalar dissipation rates in non-conservative transport systems. J Contam Hydrol 149: 46-60

Engdahl NB, Benson DA, Bolster D (2014) Predicting the enhancement of mixing-driven reactions in nonuniform flows using measures of flow topology. Phys Rev E 90: 051001

Fakhreddine S, Lee J, Kitanidis PK, Fendorf S, Rolle M (2016) Imaging geochemical heterogeneities using inverse reactive transport modeling: An example relevant for characterizing arsenic mobilization and distribution. Adv Water Resour 88: 186-197

Freyberg DL (1986) A natural gradient experiment on solute transport in a sand aquifer: 2. Spatial moments and the advection and dispersion of nonreactive tracers. Water Resour Res 22: 2031-2046

Garabedian SP, LeBlanc DR, Gelhar LW, Celia MA (1991) Large-scale natural gradient tracer test in sand and gravel, Cape Cod, Massachusetts: 2. Analysis of spatial moments for a nonreactive tracer. Water Resour Res 27: 911924

Gelhar LW, Axness CL (1983) Three-dimensional stochastic analysis of macrodispersion in aquifers. Water Resour Res 19: 161-180

Gelhar LW (1987) Stochastic analysis of solute transport in saturated and unsaturated porous media. In Advances in transport phenomena in porous media (pp. 657-700). Springer, Dordrecht.

Gramling CM, Harvey CF, Meigs LC (2002) Reactive transport in porous media: A comparison of model prediction with laboratory visualization. Environ Sci Technol 36: 2508-2514

Guedes de Carvalho JRF, Delgado JMPQ (2005) Overall map and correlation of dispersion data for flow through granular packed beds. Chem Eng Sci 60: 365-75

Haberer CM, Muniruzzaman M, Grathwohl P, Rolle M (2015) Diffusive/Dispersive and reactive fronts in porous media: Fe (II)-oxidation at the unsaturated/saturated interface. Vadose Zone J 15: 1-14

Haggerty R, Gorelick SM (1995) Multiple-rate mass transfer for modeling diffusion and surface reactions in media with pore-scale heterogeneity. Water Resour Res 31: 2383-2400

Hester ET, Bayani Cardenas M, Haggerty R, Apte SV (2017) The importance and challenge of hyporheic mixing. Water Resour Res 53: 3565-3575

Hidalgo JJ, Dentz M, Cabeza Y, Carrera J (2015) Dissolution patterns and mixing dynamics in unstable reactive flow. Geophys Res Lett 42: 6357-6364

Hochstetler DL, Kitanidis PK (2013) The behavior of effective reaction rate constants for bimolecular reactions under physical equilibrium. J Contam Hydrol 144: 88-98

Hochstetler DL, Rolle M, Chiogna G, Haberer CM, Grathwohl P, Kitanidis PK (2013) Effects of compound-specific transverse mixing on steady-state reactive plumes: Insights from pore-scale simulations and Darcy-scale experiments. Adv Water Resour 54: 1-10

Holzner M, Willmann M, Morales V, Dentz M (2015) Intermittent lagrangian velocities and accelerations in threedimensional porous medium flow. Phys Rev E 92: 013015

Jessen S, Postma D, Larsen F, Nhan PQ, Hoa LQ, Trang PTK, Long TV, Viet PH, Jakobsen R (2012) Surface complexation modeling of groundwater arsenic mobility: Results of a forced gradient experiment in a Red River flood plain aquifer, Vietnam. Geochim Cosmochim Acta 98: 186-201.

Jimenez-Martinez J, de Anna P, Tabuteau H, Turuban R, Le Borgne T, Méheust Y (2015) Pore-scale mechanisms for the enhancement of mixing in unsaturated porous media and implications for chemical reactions. Geophys Res Lett 42: 5316-5324 
Jiménez-Martínez J, Le Borgne T, Tabuteau H, Méheust Y (2017) Impact of saturation on dispersion and mixing in porous media: Photobleaching pulse injection experiments and shear-enhanced mixing model. Water Resour Res 53: 1457-1472

Kang PK, de Anna P, Nunes JP, Bijeljic B, Blunt MJ, Juanes R (2014) Pore-scale intermittent velocity structure underpinning anomalous transport through 3-D porous media. Geophys Res Lett 41: 6184-6190

Katz GE, Berkowitz B, Guadagnini A, Saaltink MW (2011) Experimental and modeling investigation of multicomponent reactive transport in porous media. J Contam Hydrol 120-121: 27-44

Kapoor V, Kitanidis PK (1996) Concentration fluctuations and dilution in two-dimensionally periodic heterogeneous porous media. Transport Porous Med 22: 91-119

Kapoor V, Gelhar L, Miralles-Wilhelm F (1997) Bimolecular second-order reactions in spatially varying flows: segregation induced scale-dependent transformation rates. Water Resour Res 33: 527-536.

Kitanidis PK (1992) Analysis of macrodispersion through volume-averaging - moment equations. Stoch Hydrol Hydraul 6: 5-25

Kitanidis PK (1994) The concept of the dilution index. Water Resour Res 30: 2011-2026.

Kitanidis PK (2015) Persistent questions of heterogeneity, uncertainty, and scales in subsurface flow and transport. Water Resour Res 51: 5888-5904

Kitanidis PK (2017) Teaching and communicating dispersion in hydrogeology, with emphasis on the applicability of the Fickian model. Adv Water Resour 106: 11-23

Kitanidis PK, McCarty PL (2012) Delivery and mixing in the subsurface: Processes and design principles for in situ remediation. Springer, New York, USA

Knutson C, Valocchi AJ, Werth CJ (2007) Comparison of continuum and pore-scale models of nutrient biodegradation under transverse mixing conditions. Adv Water Resour 30: 1421-1431

Kocar BD, Polizzotto ML, Benner SG, Ying SC, Ung M, Ouch K, Samreth S, Suy B, Phan K., Sampson M, Fendorf S (2008) Integrated biogeochemical and hydrologic processes driving arsenic release from shallow sediments to groundwaters of the Mekong delta. Appl Geochem 23: 3059-3071

Kocar B, Herbel M, Tufano K., Fendorf S (2006) Contrasting effects of dissimilatory iron (III) and arsenic (V) reduction on arsenic retention and transport. Environ Sci Technol 40: 6715-6721

Larralde H, Araujo M, Havlin S, Stanley HE (1992) Reaction front for A + B $\rightarrow$ C diffusion-reaction systems with initially separated reactants. Phys Rev A 46: 855-859

Le Borgne T, Dentz M, Bolster D, Carrera J, De Dreuzy J, Davy P (2010) Non-Fickian mixing: Temporal evolution of the scalar dissipation rate in heterogeneous porous media. Adv Water Resour 33: 1468-1475

Le Borgne T, Dentz M, Davy P, Bolster D, de Dreuzy JR, Bour O (2011) Persistence of incomplete mixing: A key to anomalous transport. Phys Rev E 84: 015301(R)

Le Borgne T, Ginn T, Dentz M (2014) Impact of fluid deformation on mixing-induced chemical reactions in heterogeneous flows. Geophys Res Lett 41: 7898-7906

Le Borgne T, Dentz M, Villermaux E (2015) The lamellar description of mixing in porous media. J Fluid Mech 770: 458-498.

Lee J, Rolle M, Kitanidis PK (2018) Longitudinal dispersion coefficients for numerical modeling of solute transport in heterogeneous formations. J Contam Hydrol 212: 41-54

Lester DR, Metcalfe G, Trefry MG (2013) Is chaotic advection inherent to porous media flow? Phys Rev Lett 111: 174101

Lester DR, Dentz M, Le Borgne T (2016) Chaotic mixing in three-dimensional porous media. J Fluid Mech 803: 144174

Li L, Steefel CI, Yang L (2008) Scale dependence of mineral dissolution rates within single pores and fractures. Geochim Cosmochim Acta, 72: 360-377 
Li L et al. (2017) Expanding the role of reactive transport models in critical zone processes. Earth Sci Rev 165: 280301

Loyaux-Lawniczak S, Lehmann F, Ackerer P (2012) Acid/base front propagation in saturated porous media: 2D laboratory experiments and modeling. J Contam Hydrol 138-139: 15-21

Luo J, Dentz M, Carrera J, Kitanidis PK (2008) Effective reaction parameters for mixing-controlled reactions in heterogeneous media. Water Resour Res 44: W02416

Ma R, Zheng C, Prommer H, Greskowiak J, Liu C, Zachara J, Rochold M (2010) A field-scale reactive transport model for $\mathrm{U}(\mathrm{VI})$ migration influenced by coupled multirate mass transfer and surface complexation reactions. Water Resour Res 46: W05509

Martinez-Ruiz D, Meunier P, Favier B, Duchemin L, Villermaux E (2018) The diffusive sheet method for scalar mixing. J Fluid Mech 837: 230-257

Martinez-Landa L, Carrera J, Dentz M, Fernandez-Garcia D, Nardi A, Saaltink M (2012) Mixing induced reactive transport in fractured crystalline rocks. Appl Geochem 27: 479-489

Mays DC, Neupauer R (2012) Plume spreading in groundwater by stretching and folding. Water Resour Res 48 : W07501

McAllister SM, Barnett JM, Heiss JW, Findlay AJ, MacDonald DJ, Dow CL, Luther GW, Michael HA, Chan CS (2015) Dynamic hydrologic and biogeochemical processes drive microbially enhanced iron and sulfur cycling within the intertidal mixing zone of a beach aquifer. Limnol and Oceanogr 60: 329-345

McClain, M et al. (2003) Biogeochemical hot spots and hot moments at the interface of terrestrial and aquatic ecosystems. Ecosystems 6: 301-312

McMahon P (2001) Aquifer/aquitard interfaces: Mixing zones that enhance biogeochemical reactions. Hydrogeol J 9: $34-43$

Meile C, Tuncay K (2006) Scale dependence of reaction rates in porous media. Adv Water Resour 29: 62-71

Molins S (20XX) Multiscale reactive transport approaches. Rev Mineral Geochem XX:xxx-xxx

Molins S, Trebotich D, Steefel C, Shen C (2012) An investigation of the effect of pore scale flow on average geochemical reaction rates using direct numerical simulation. Water Resour Res 48: W03527

Muniruzzaman M, Rolle M (2015) Impact of multicomponent ionic transport on $\mathrm{pH}$ fronts propagation in saturated porous media. Water Resour Res 51: 6739-6755

Muniruzzaman M, Rolle M (2016) Modeling multicomponent ionic transport in groundwater with IPhreeqc coupling: Electrostatic interactions and geochemical reactions in homogeneous and heterogeneous domains. Adv Water Resour 98: 1-15

Muniruzzaman M, Rolle M (2017) Experimental investigation of the impact of compound-specific dispersion and electrostatic interactions on transient transport and solute breakthrough. Water Resour Res 53: 1189-1209

Muniruzzaman M, Haberer CM, Grathwohl P, Rolle M (2014) Multicomponent ionic dispersion during transport of electrolytes in heterogeneous porous media: Experiments and model-based interpretation. Geochim Cosmochim Acta 141: 656-669

Ottino JM (1989) The kinematics of mixing: stretching, chaos, and transport. Cambridge University Press

Paster A, Aquino T, Bolster D (2015) Incomplete mixing and reactions in laminar shear flow. Phys Rev E 92: 012922

Piscopo AN, Neupauer RM, Mays DC (2013) Engineered injection and extraction to enhance reaction for improved in situ remediation. Water Resour Res 49: 3618-3625

Pool M, Dentz M (2018) Effects of heterogeneity, connectivity and density variations on mixing and chemical reactions under temporally fluctuating flow conditions and the formation of reaction patterns. Water Resour Res 54: $186-204$

Poonoosamy J, Kosakowski G, Van Loon L, Mäder U (2015) Dissolution-precipitation processes in tank experiments for testing numerical models for reactive transport calculations: Experiments and modelling. J Contam Hydrol 177-178: $1-17$ 
Prommer H, Tuxen N, Bjerg PL (2006) Fringe-controlled natural attenuation of phenoxy acids in a landfill plume: Integration of field-scale processes by reactive transport modeling. Environ Sci Technol 40: 4732-4738

Prommer H, Anneser B, Rolle M, Einsiedl F, Griebler C (2009) Biogeochemical and isotopic gradients in a BTEX/PAH contaminant plume: model-based interpretation of a high-resolution field data set. Environ Sci Technol 43: 8206-8212

Ranz WE (1979) Applications of a stretch model to mixing, diffusion, and reaction in laminar and turbulent flows. AIChE J 25: 41-47

Rasa E, Chapman SW, Bekins BA, Fogg GE, Scow KM, Mackay DM (2011) Role of back diffusion and biodegradation reactions in sustaining an MTBE/TBA plume in alluvial media. J Contam Hydrol 126: 235-247

Rasouli P, Steefel CI, Mayer UK, Rolle M (2015) Benchmarks for multicomponent diffusion and electrochemical migration. Computat Geosci 19: 523-533

Rehfeldt KR, Boggs JM, Gelhar LW (1992) Field study of dispersion in an heterogeneous aquifer 3. geostatistical analysis of hydraulic conductivity. Water Resour Res 28: 3309-3324

Rolle M, Kitanidis PK (2014) Effects of compound-specific dilution on transient transport and solute breakthrough: A pore-scale analysis. Adv Water Resour 71: 186-199

Rolle M, Clement TP, Sethi R, Di Molfetta A (2008) A kinetic approach for simulating redox-controlled fringe and core biodegradation processes in groundwater: model development and application to a landfill site in Piedmont, Italy. Hydrol Process 22: 4905-4921

Rolle M, Eberhardt C, Chiogna G, Cirpka OA, Grathwohl P (2009) Enhancement of dilution and transverse reactive mixing in porous media: Experiments and model-based interpretation. J Contam Hydrol 110: 130-142

Rolle M, Chiogna G, Bauer R, Griebler C, Grathwohl P (2010) Isotopic fractionation by transverse dispersion: flowthrough microcosms and reactive transport modeling study. Environ Sci Technol 44: 6167-6173

Rolle M, Hochstetler DL, Chiogna G, Kitanidis PK, Grathwohl P (2012) Experimental investigation and pore-scale modeling interpretation of compound-specific transverse dispersion in porous media. Transport Porous Med 93: 347-362

Rolle M, Chiogna G, Hochstetler DL, Kitanidis PK (2013a) On the importance of diffusion and compound-specific mixing for groundwater transport: An investigation from pore to field scale. J Contam Hydrol 153: 51-68

Rolle M, Muniruzzaman M, Haberer CM, Grathwohl P (2013b) Coulombic effects in advection-dominated transport of electrolytes in porous media: Multicomponent ionic dispersion. Geochim Cosmochim Acta 120: 195-205, doi: 10.1016/j.gca.2013.06.031

Rolle M, Sprocati R, Masi M, Jin B, Muniruzzaman M (2018) Nernst-Planck based description of transport, Coulombic interactions and geochemical reactions in porous media: Modeling approach and benchmark experiments. Water Resour Res 54: 3176-3195

Rubin Y (2003) Applied Stochastic Hydrogeology, Oxford Univ. Press, Oxford, UK

Rubin Y, Sun A, Maxwell R, Bellin A (1999) The concept of block-effective macrodispersivity and a unified approach for grid-scale- and plume-scale-dependent transport. J Fluid Mech 395: 161-180

Scheibe TD, Schuchardt K, Agarwal K, Chase J, Yang X, Palmer BJ, Tartakovsky AM, Elsethagen T, Redden G (2015) Hybrid multiscale simulation of a mixing-controlled reaction. Adv Water Resour 83: 228-239

Scheidegger AE (1961) General theory of dispersion in porous media. J Geophys Res 66: 3273-3278

Semprini L, Roberts PV, Hopkins GD, McCarty PL (1990) Field evaluation of in situ biodegradation of chlorinated ethenes: Part 2, Results of biostimulation and biotransformation experiments. Ground Water 28: 715-727

Song X, Hong E, Seagren EA (2014) Laboratory-scale in situ bioremediation in heterogeneous porous media: Biokinetics-limited scenario. J Contam Hydrol 158: 78-92

Souzy M, Zaier I, Lhuissier H, Le Borgne T, Metzger B. (2018) Mixing lamellae in a shear flow. J Fluid Mech 838: R3-1-R3-12

Steefel CI, Appelo CAJ, Arora B, Jacques D, Kalbacher T, Kolditz O, Yeh GT (2015) Reactive transport codes for subsurface environmental simulation. Computat Geosci, 19: 445-78 
Stegen JC et al. (2015) Groundwater-surface water mixing shifts ecological assembly processes and stimulates organic carbon turnover. Nat Commun 7: 11237

Stolze L, Zhang D, Guo H, Rolle M (2019) Surface complexation modeling of arsenic mobilization from goethite: Interpretation of an in-situ experiment. Geochim Cosmochim Acta 248: 274-288

Sudicky EA, Illman WA (2011) Lessons learned from a suite of CFB Borden experiments. Ground Water 49: 630648

Stauffer F (2007) Impact of highly permeable sediment units with inclined bedding on solute transport in aquifers. Adv Water Res 30: 2194-2201

Tartakovsky AM, Redden GD, Lichtner PC, Scheibe TC, Meakin P (2008) Mixing-induced precipitation: Experimental study and multi-scale numerical analysis. Water Resour Res 44: W06S04

Tartakovsky GD, Tartakovsky AM, Scheibe TD, Fang Y, Mahadevan R, Lovley DR (2013) Pore-scale simulation of microbial growth using a genome-scale metabolic model: Implications for Darcy-scale reactive transport. Adv Water Resour 59: 256-270

Thullner M, Mauclaire L, Schroth MH, Kinzelbach W, Zeyer J (2002) Interaction between water flow and spatial distribution of microbial growth in a two-dimensional flow field in saturated porous media. J Contam Hydrol 58: $169-189$

Thullner M, Van Cappellen P, Regnier P (2005) Modeling the impact of microbial activity on redox dynamics in porous media. Geochim Cosmochim Acta 69: 5005-5019

Toth, J. (1963) A theoretical analysis of groundwater ow in small drainage basins, J Geophys Res 68: 4795-4812

Tournassat C, Steefel CI (2015) Ionic transport in nano-porous clays with consideration of electrostatic effects. Rev Mineral Geochem 80: 287-329

Trefry MG, Lester DR, Metcalfe G, Ord A, Regenauer-Lieb K (2012) Toward enhanced subsurface intervention methods using chaotic advection. J Contam Hydrol 127: 15-29

Turuban R, Lester DR, Le Borgne T, Méheust Y (2018) Space-group symmetries generate chaotic fluid advection in crystalline granular media. Phys Rev Lett 120: 024501

Tuxen N, Albrechtsen H-J, Bjerg PL (2006) Identification of a reactive degradation zone at a landfill leachate plume fringe using high resolution sampling and incubation techniques. J Contam Hydrol 85: 179-194

Valocchi AJ, Bolster D, Werth CJ (2018) Mixing-limited reactions in porous media. Transport Porous Med 126: 1-26

Van Breukelen BM, Rolle M (2012) Transverse hydrodynamic dispersion effects on isotope signals in groundwater chlorinated solvents' plumes. Environ Sci Technol 46: 7700-7708

Villermaux E (2019) Mixing Versus Stirring, Annu. Rev Fluid Mech 51: 245-273

Wang J, Kitanidis PK (1999) Analysis of macrodispersion through volume averaging: comparison with stochastic theory. Stochastic Environ Res Risk Assess 13: 66-84

Werth CJ, Cirpka OA, Grathwohl P (2006) Enhanced mixing and reaction through flow focusing in heterogeneous porous media. Water Resour Res 42: W12414

Whitaker (1999) The method of volume averaging. Springer

Willingham TW, Werth CJ, Valocchi AJ (2008) Evaluation of the effects of porous media structure on mixingcontrolled reactions using pore-scale modeling and micromodel experiments. Environ Sci Technol 42: 31853193

Winderl C, Anneser B, Griebler C, Meckenstock RU, Lueders T (2008) Depth-resolved quantification of anaerobic toluene degraders and aquifer microbial community patterns in distinct redox zones of a tar oil contaminant plume. Appl Environ Microbiol 74: 792-801

Wright EE, Richter DH, Bolster D (2017) Effects of incomplete mixing on reactive transport in flows through heterogeneous porous media. Phys Rev Fluids 2: 114501 
Ye Y, Chiogna G, Cirpka OA, Grathwohl P, Rolle M (2015a) Experimental investigation of compound-specific dilution of solute plumes in saturated porous media: 2-D vs. 3-D flow-through systems. J Contam Hydrol 172: $33-47$

Ye Y, Chiogna G, Cirpka OA, Grathwohl P, Rolle M (2015b). Enhancement of plume dilution in three-dimensional porous media by flow-focusing in high-permeability inclusions. Water Resour Res 51: 5582-5602

Ye Y., Chiogna G, Cirpka OA, Grathwohl P, Rolle M (2015c) Experimental evidence of helical flow in porous media. Phys Rev Lett 115: 194502

Ye Y, Chiogna G, Cirpka OA, Grathwohl P, Rolle M (2016) Experimental investigation of transverse mixing in porous media under helical flow conditions. Phys Rev E 94: 013113

Ye Y, Chiogna G, Lu C, Rolle M (2018) Effect of anisotropy on plume entropy and reactive mixing in helical flows. Transp Porous Med 121: 315-332

Yoon H, Valocchi A, Werth C, Dewers T (2012) Pore-scale simulation of mixing-induced calcium carbonate precipitation and dissolution in a microfluidic pore network. Water Resour Res 48: W02524

Zhang C, Kang Q, Wang X, Zilles JL, Mueller RH, Werth CJ (2010a) Effects of pore-scale heterogeneity and transverse mixing on bacterial growth in porous media. Environ Sci Technol 44: 3085-3092

Zhang C, Dehoff K, Hess N, Oostrom M, Wietsma TW, Valocchi AJ, Fouke BW, Werth C (2010b) Pore-scale study of transverse mixing induced $\mathrm{CaCO}_{3}$ precipitation and permeability reduction in a model subsurface sedimentary system. Environ Sci Technol 44: 7833-7838

Zheng C, Bianchi M, Gorelick S (2011) Lessons learned from 25 years of research at the MADE site. Ground Water 49: 649-662 